\title{
Dirac operators on all Podleś quantum spheres
}

\author{
Ludwik Dąbrowski, Francesco D’Andrea, Giovanni Landi, and Elmar Wagner
}

\begin{abstract}
We construct spectral triples on all Podleś quantum spheres $\mathrm{S}_{q t}^{2}$. These noncommutative geometries are equivariant for a left action of $U_{q}(\mathrm{su}(2))$ and are regular, even and of metric dimension 2. They are all isospectral to the undeformed round geometry of the sphere $S^{2}$. There is also an equivariant real structure for which both the commutant property and the first order condition for the Dirac operators are valid up to infinitesimals of arbitrary order.
\end{abstract}

Mathematics Subject Classification (2000). 58B34, $17 \mathrm{~B} 37$.

Keywords. Noncommutative geometry, spectral triples, quantum groups, quantum spheres.

\section{Introduction}

We report on further explorations of the land where noncommutative geometry meets quantum groups and describe even spectral triples $\left(\mathcal{A}\left(\mathrm{S}_{q t}^{2}\right), \mathscr{H}, D, \gamma\right)$ on all the quantum 2-spheres $\mathcal{A}\left(\mathrm{S}_{q t}^{2}\right)$ introduced by Podleś [12]. Here the real deformation parameter is taken to be in the interval $0<q<1$. The additional parameter $t \in[0,1]$ labels, among other things, the classical points, i.e., the 1 dimensional representations of the algebra $\mathcal{A}\left(\mathrm{S}_{q t}^{2}\right)$.

The spectral triples of the present paper generalize the 0-dimensional one of [7] for the standard 2-sphere and the isospectral 2-dimensional one of [5] for the equatorial 2-sphere. In particular, we have spectral triples of metric dimension 2 on all Podleś quantum 2-spheres with the eigenvalues of the Dirac operator depending linearly on an angular momentum label (and with the 'correct' multiplicities). The Dirac operators have the same spectrum as the Dirac operator of the 'round' metric on the usual 2sphere and we are constructing isospectral deformations. Moreover, all these triples are regular with simple and discrete dimension spectrum $\Sigma=\{1,2\}$, as expected from the commutative case. On the standard sphere we have additional families of spectral triples with eigenvalues of $|D|$ growing not faster than $q^{-l}$ for large $l$.

There is a crucial equivariance of the representation of the algebra $\mathcal{A}\left(\mathrm{S}_{q t}^{2}\right)$ on the Hilbert space of spinors $\mathscr{H}$ and of the Dirac operator $D$ under a left action of the quantum enveloping algebra $U_{q}(\operatorname{su}(2))$. We have also an equivariant real structure 
of the kind 'up to compact operators' of [5], that is, such that both the 'commutant property' and the 'first order condition' for a real spectral triple [3] are obeyed only up to infinitesimals of arbitrary order. This phenomenon has also been observed in [6] for the manifold of $\mathcal{A}\left(\mathrm{SU}_{q}(2)\right)$. Its occurrence for all Podleś quantum 2 -spheres is another indication that it may be a characteristic feature of noncommutative manifolds of quantum groups and associated quantum (homogeneous) spaces, at least when asking for some degree of equivariance. For the standard sphere, with the eigenvalues of $|D|$ behaving like $\left|d_{l}\right| \sim q^{-l}$, the conditions on the real structure need not be modified [7].

It should be mentioned that we do not study the additional 'discrete' series of quantum 2-spheres in [12] which have algebras of matrices as coordinate algebras.

\section{Preliminary definitions}

In this section, we set out the basic notions concerning equivariant spectral triples. We start by recalling the notion of a finite summable spectral triple [2].

Definition 2.1. A spectral triple $(\mathcal{A}, \mathscr{H}, D)$ is given by a complex unital $*$-algebra $\mathcal{A}$, a faithful $*$-representation $\pi$ of $\mathcal{A}$ by bounded operators on a (separable) Hilbert space $\mathscr{H}$, and a self-adjoint operator $D$ (the Dirac operator) such that

(i) $(D+i)^{-1}$ is a compact operator,

(ii) $[D, \pi(a)]$ is bounded for all $a \in \mathcal{A}$.

With $0<\mu<\infty$, the spectral triple is said to be $\mu^{+}$-summable if the operator $\left(D^{2}+1\right)^{-1 / 2}$ is in the Dixmier ideal $\mathscr{L}^{\mu+}(\mathscr{H})$.

We shall also call $\mu$ the metric dimension of the triple.

A spectral triple is called even if there exists a $\mathbb{Z}_{2}$-grading operator $\gamma$ on $\mathscr{H}$, $\gamma=\gamma^{*}, \gamma^{2}=1$, such that the Dirac operator is odd and the algebra is even, i.e.,

$$
\gamma D=-D \gamma, \quad \pi(a) \gamma=\gamma \pi(a), \quad a \in \mathcal{A} .
$$

Recall [3] that a real structure on a spectral triple $(\mathcal{A}, \mathscr{H}, D)$ should be given by an antiunitary operator $J$ on $\mathscr{H}$ fulfilling the conditions $J^{2}= \pm 1, J D= \pm D J$ and

$$
\left[\pi(a), J \pi(b) J^{-1}\right]=0, \quad\left[[D, \pi(a)], J \pi(b) J^{-1}\right]=0, \quad a, b \in \mathcal{A} .
$$

It was suggested in [5] that one should modify these conditions in order to obtain a nontrivial spin geometry on the coordinate algebra of quantum groups and of associated quantum (homogeneous) spaces. Following the lines of [5], we impose the weaker assumption that (2.2) holds only modulo infinitesimals of arbitrary high order. Here, a compact operator $A$ is regarded as an infinitesimal of arbitrary high order if its singular values $s_{k}(A)$ satisfy $\lim _{k \rightarrow \infty} k^{p} s_{k}(A)=0$ for all $p>0$. Therefore, throughout this paper, we shall use the following working definition of a real structure. 
Definition 2.2. A real structure $J$ on a spectral triple $(\mathcal{A}, \mathscr{H}, D)$ is given by an antiunitary operator $J$ on $\mathscr{H}$ such that

$$
\begin{gathered}
J^{2}= \pm 1, \quad J D= \pm D J \\
{\left[\pi(a), J \pi(b) J^{-1}\right] \in \mathcal{L}, \quad\left[[D, \pi(a)], J \pi(b) J^{-1}\right] \in \mathcal{d}, \quad a, b \in \mathcal{A},}
\end{gathered}
$$

where $d$ is an operator ideal of infinitesimals of arbitrary high order. We shall name the datum $(\mathcal{A}, \mathscr{H}, D, J)$ a real spectral triple (up to infinitesimals).

If $(\mathcal{A}, \mathscr{H}, D, \gamma)$ is even and if in addition

$$
J \gamma= \pm \gamma J
$$

we call the datum $(\mathcal{A}, \mathcal{H}, D, \gamma, J)$ a real even spectral triple (up to infinitesimals).

The signs above depend on the (metric) dimension. We are only interested in the case when the dimension is 2 . Then $J^{2}=-1, J D=D J$ and $J \gamma=-\gamma J$. For brevity, we shall drop the annotation "up to infinitesimals" in the sequel.

We turn now to symmetries that will be implemented by an action of a Hopf $*$ algebra. In the classical case of a $G$-homogeneous $\operatorname{spin}^{c}$ structure on a manifold $M$, the symmetry Hopf $*$-algebra is given by the universal enveloping algebra $U(\mathrm{~g})$ of the Lie algebra $g$ of $G$. As we shall see, this approach will force us to consider unbounded *-representations. For this, let $\mathcal{V}$ be a dense linear subspace of a Hilbert space $\mathscr{H}$ with inner product $\langle\cdot, \cdot\rangle$, and let $U$ be a $*$-algebra. An (unbounded) $*$-representation of $\mathcal{U}$ on $\mathcal{V}$ is a homomorphism $\lambda: \mathcal{U} \rightarrow \operatorname{End}(\mathcal{V})$ such that $\langle\lambda(h) v, w\rangle=\left\langle v, \lambda\left(h^{*}\right) w\right\rangle$ for all $v, w \in \mathcal{V}$ and all $h \in \mathcal{U}$.

Next let $\mathcal{U}=(\mathcal{U}, \Delta, S, \varepsilon)$ be a Hopf $*$-algebra and $\mathcal{A}$ be a left $\mathcal{U}$-module $*$ algebra, i.e., there is a left action $\triangleright$ of a $\mathcal{U}$ on $\mathcal{A}$ satisfying

$$
h \triangleright x y=\left(h_{(1)} \triangleright x\right)\left(h_{(2)} \triangleright y\right), \quad h \triangleright 1=\varepsilon(h) 1, \quad(h \triangleright x)^{*}=S(h)^{*} \triangleright x^{*}
$$

for all $h \in \mathcal{U}$ and $x, y \in \mathcal{A}$. As customary, we use the notation $\Delta(h)=h_{(1)} \otimes h_{(2)}$.

A $*$-representation $\pi$ of $\mathcal{A}$ on $\mathcal{V}$ is called $U$-equivariant if there exists a $*$-representation $\lambda$ of $U$ on $\mathcal{V}$ such that

$$
\lambda(h) \pi(x) \xi=\pi\left(h_{(1)} \triangleright x\right) \lambda\left(h_{(2)}\right) \xi,
$$

for all $h \in \mathcal{U}, x \in \mathcal{A}$ and $\xi \in \mathcal{V}$. Given $\mathcal{U}$ and $\mathcal{A}$ as above, the left crossed product $*$-algebra $\mathcal{A} \rtimes \mathcal{U}$ is defined as the $*$-algebra generated by the two $*$-subalgebras $\mathcal{A}$ and $\mathcal{U}$ with crossed commutation relations

$$
h x=\left(h_{(1)} \triangleright x\right) h_{(2)}, \quad h \in \mathcal{U}, x \in \mathcal{A} .
$$

Thus $\mathcal{U}$-equivariant representations of $\mathcal{A}$ correspond to $*$-representations of $\mathcal{A} \rtimes \mathcal{U}$. 
A linear operator $D$ defined on $\mathcal{V}$ is said to be equivariant if it commutes with $\lambda(h)$, i.e.,

$$
D \lambda(h) \xi=\lambda(h) D \xi,
$$

for all $h \in \mathcal{U}$ and $\xi \in \mathcal{V}$. On the other hand, an antiunitary operator $J$ is said to be equivariant if it leaves $\mathcal{V}$ invariant and if it is the antiunitary part in the polar decomposition of an antilinear (closed) operator $T$ that satisfies the condition

$$
T \lambda(h) \xi=\lambda\left(S(h)^{*}\right) T \xi
$$

for all $h \in \mathcal{U}$ and $\xi \in \mathcal{V}$, where $S$ denotes the antipode of $\mathcal{U}$.

We collect all these equivariance requirements by giving the following definition [14].

Definition 2.3. Let $\mathcal{U}$ be a Hopf $*$-algebra, $\mathcal{A}$ a left $\mathcal{U}$-module $*$-algebra and $\pi$ a $\mathcal{U}$-equivariant representation of $\mathcal{A}$ on (a dense linear subspace of) a Hilbert space $\mathscr{H}$. A (real even) spectral triple $(\mathcal{A}, \mathscr{H}, D, \gamma, J)$ is called equivariant if the operators $D$, $\gamma$ and $J$ are equivariant in the above sense.

In the remainder of this section, we recall a few analytic properties of spectral triples [4]. With the operator $D$ of a spectral triple $(\mathscr{A}, \mathcal{H}, D)$, one defines an unbounded derivation $\delta$ on $\mathscr{B}(\mathcal{H})$ by

$$
\delta(a)=[|D|, a], \quad a \in \mathscr{B}(\mathscr{H}) .
$$

Definition 2.4. The spectral triple $(\mathcal{A}, \mathscr{H}, D)$ is said to be regular if the algebra generated by $\mathcal{A}$ and the commutators $[D, \mathcal{A}]$ belongs to

$$
\mathrm{OP}^{0}:=\bigcap_{j \in \mathbb{N}_{0}} \operatorname{dom} \delta^{j} .
$$

For a regular spectral triple, the algebra $\Psi^{0}$ generated by $\mathcal{A}$, the commutators $[D, \mathcal{A}]$ and iterated applications of $\delta$ is a subalgebra of $\mathrm{OP}^{0}, \Psi^{0} \subset \mathrm{OP}^{0}$. If the spectral triple is of dimension $\mu$, the "zeta-type" functions

$$
\zeta_{a}(z):=\operatorname{Tr}_{\mathscr{H}}\left(a|D|^{-z}\right), \quad a \in \Psi^{0},
$$

are defined and holomorphic for $z \in \mathbb{C}$ with $\operatorname{Re} z>\mu$. Here we are assuming, for simplicity, that $D$ is invertible. Analogous formulae are easily defined for the general case: one works with $\left(1+D^{2}\right)^{-z / 2}$ instead of $|D|^{-z}$ for a not invertible $D$.

Definition 2.5. A spectral triple has dimension spectrum $\Sigma \subset \mathbb{C}$, with $\Sigma$ a countable set, if all $\zeta_{a}(z), a \in \Psi^{0}$, extend to meromorphic functions on $\mathbb{C}$ with poles in $\Sigma$ as unique singularities. 
For later use, we also recall the definition of "smoothing operators" $\mathrm{OP}^{-\infty}$, i.e.,

$$
\mathrm{OP}^{-\infty}:=\left\{T \in \mathrm{OP}^{0}:|D|^{n} T \in \mathrm{OP}^{0} \text { for all } n \in \mathbb{N}_{0}\right\} .
$$

The class $\mathrm{OP}^{-\infty}$ is a two-sided $*$-ideal in the $*$-algebra $\mathrm{OP}^{0}$ and $\delta$-invariant. When computing the dimension spectrum, we can take the quotient by smoothing operators since they merely contribute holomorphic terms.

If the metric dimension of $D$ is finite, smoothing operators are infinitesimals of arbitrary high order. But the converse is in general not true, since infinitesimals of arbitrary high order are a two-sided $*$-ideal in $\mathscr{B}(\mathscr{H})$ while $\mathrm{OP}^{-\infty}$ is not.

Also, for a finite metric dimension, rapid decay matrices (in a basis of eigenvectors for $D$ with eigenvalues in increasing order) are smoothing operators.

\section{The Podleś spheres and their symmetries}

Recall that $q \in(0,1)$ and $t \in[0,1]$. Let

$$
[n]=[n]_{q}:=\frac{q^{n}-q^{-n}}{q-q^{-1}}, \quad n \in \mathbb{R} .
$$

The coordinate $*$-algebra $\mathcal{A}\left(\mathrm{S}_{q t}^{2}\right)$ of the Podleś spheres [12] is generated by elements $x_{-1}, x_{0}$ and $x_{1}$ with relations

$$
\begin{gathered}
x_{-1}\left(x_{0}-t\right)=q^{2}\left(x_{0}-t\right) x_{-1}, \\
x_{1}\left(x_{0}-t\right)=q^{-2}\left(x_{0}-t\right) x_{1}, \\
-[2] x_{-1} x_{1}+\left(q^{2} x_{0}+t\right)\left(x_{0}-t\right)=[2]^{2}(1-t), \\
-[2] x_{1} x_{-1}+\left(q^{-2} x_{0}+t\right)\left(x_{0}-t\right)=[2]^{2}(1-t),
\end{gathered}
$$

and with involution $x_{-1}^{*}=-q^{-1} x_{1}$ and $x_{0}^{*}=x_{0}$.

The standard quantum sphere corresponds to the value $t=1$, the equatorial one to $t=0$. For $t=1$, the quantum sphere has one classical point. That is, there exists exactly one 1-dimensional irreducible representation of the algebra, namely,

$$
x_{0}=1, \quad x_{1}=x_{-1}=0 .
$$

For $t \neq 1$, the classical points make up an $\mathrm{S}^{1}$ given by the following 1-dimensional irreducible representations of the algebra,

$$
x_{0}=t, \quad x_{1}=\sqrt{\left(1+q^{2}\right)(1-t)} \lambda, \quad x_{-1}=-q^{-1} \sqrt{\left(1+q^{2}\right)(1-t)} \bar{\lambda},
$$

where $\lambda$ is a complex number of modulus 1 . 
The generators that we use are related to those of [12] as follows. For $t \neq 0$, let $c=t^{-1}-t$. Setting

$$
A=\left(1+q^{2}\right)^{-1}\left(1-t^{-1} x_{0}\right), \quad B=q\left(1+q^{2}\right)^{-1 / 2} t^{-1} x_{-1},
$$

we recover the generators from [12], equation (7a), satisfying the relations

$A B=q^{-2} B A, \quad A B^{*}=q^{2} B^{*} A, \quad B^{*} B=A-A^{2}+c, \quad B B^{*}=q^{2} A-q^{4} A^{2}+c$.

For $t=0$, we set

$$
A=-\left(1+q^{2}\right)^{-1} x_{0}, \quad B=q\left(1+q^{2}\right)^{-1 / 2} x_{-1},
$$

and obtain the generators from [12], equation (7b), with relations

$$
A B=q^{-2} B A, \quad A B^{*}=q^{2} B^{*} A, \quad B^{*} B=-A^{2}+1, \quad B B^{*}=-q^{4} A^{2}+1 .
$$

The symmetry that we shall use in the following is given by the Hopf $*$-algebra $U_{q}(\operatorname{su}(2))$. This Hopf $*$-algebra is generated by elements $f, e, k, k^{-1}$ with defining relations (cf. [10])

$k k^{-1}=k^{-1} k=1, k^{2}-k^{-2}=\left(q-q^{-1}\right)(f e-e f), k f=q f k, k e=q^{-1} e k$,

coproduct

$$
\Delta k=k \otimes k, \quad \Delta f=f \otimes k+k^{-1} \otimes f, \quad \Delta e=e \otimes k+k^{-1} \otimes e,
$$

counit

$$
\varepsilon(k-1)=\varepsilon(f)=\varepsilon(e)=0,
$$

antipode

$$
S(k)=k^{-1}, \quad S(f)=-q f, \quad S(e)=-q^{-1} e,
$$

and involution

$$
k^{*}=k, \quad f^{*}=e .
$$

The irreducible finite dimensional $*$-representations of $U_{q}(\operatorname{su}(2))$ are labeled by non-negative half-integers $l \in \frac{1}{2} \mathbb{N}_{0}$ called spin. The $\operatorname{spin} l$ representation, say $\sigma_{l}$, acts on a Hilbert space $\mathcal{V}^{l}$ which is $(2 l+1)$-dimensional; the action of the generators on an orthonormal basis $\left\{v_{m}^{l}: m=-l,-l+1, \ldots, l\right\}$ is given by the formulae,

$$
\begin{aligned}
\sigma_{l}(k) v_{m}^{l} & =q^{m} v_{m}^{l}, \\
\sigma_{l}(f) v_{m}^{l} & =[l-m]^{1 / 2}[l+m+1]^{1 / 2} v_{m+1}^{l}, \\
\sigma_{l}(e) v_{m}^{l} & =[l-m+1]^{1 / 2}[l+m]^{1 / 2} v_{m-1}^{l} .
\end{aligned}
$$


Finally, there is a left $\mathcal{U}_{q}(\mathrm{su}(2))$-action on $\mathcal{A}\left(\mathrm{S}_{q t}^{2}\right)$ which turns the latter into a $u_{q}(\mathrm{su}(2))$-module $*$-algebra. On generators, it is given by

$$
\begin{array}{lll}
k \triangleright x_{-1}=q^{-1} x_{-1}, & f \triangleright x_{-1}=[2]^{1 / 2} x_{0}, & e \triangleright x_{-1}=0, \\
k \triangleright x_{0}=x_{0}, & f \triangleright x_{0}=[2]^{1 / 2} x_{1}, & e \triangleright x_{0}=[2]^{1 / 2} x_{-1}, \\
k \triangleright x_{1}=q x_{1}, & f \triangleright x_{1}=0, & e \triangleright x_{1}=[2]^{1 / 2} x_{0} .
\end{array}
$$

Note that the generators $x_{m}, m=-1,0,+1$, of $\mathcal{A}\left(\mathrm{S}_{q t}^{2}\right)$ transform like the basis vectors $v_{m}^{1}$ of the representation $\sigma_{1}$.

\section{Equivariant representations}

As mentioned above, looking for $U_{q}(\operatorname{su}(2))$-equivariant representation of the algebra $\mathcal{A}\left(\mathrm{S}_{q t}^{2}\right)$, we can equivalently look for representations of the crossed product algebra $\mathcal{A}\left(\mathrm{S}_{q t}^{2}\right) \rtimes \mathcal{U}_{q}(\mathrm{su}(2))$; these were constructed in [13].

4.1. Integrable representations of $\mathcal{A}\left(\mathbf{S}_{q t}^{2}\right) \times \boldsymbol{U}_{q}(\operatorname{su}(2))$. A *-representation of $\mathcal{A}\left(\mathrm{S}_{q t}^{2}\right) \rtimes U_{q}(\mathrm{su}(2))$ is called integrable if its restriction to $U_{q}(\mathrm{su}(2))$ is a direct sum of $\operatorname{spin} l$ representations $\sigma_{l}$.

The integrable representations of $\mathcal{A}\left(\mathrm{S}_{q t}^{2}\right) \rtimes \mathcal{U}_{q}(\mathrm{su}(2))$ were completely classified in [13]. It turned out that each such integrable representation is a direct sum of irreducible ones. The following proposition restates Theorem 4.1 in [13] in the case of irreducible representations. In doing so, we replace the parameter $r$ there by $t^{-1}-t$.

Proposition 4.1. Let $N \in \frac{1}{2} \mathbb{Z}$. Any irreducible integrable representation of the *-algebra $\mathcal{A}\left(\mathrm{S}_{q t}^{2}\right) \rtimes \mathcal{U}_{q}(\mathrm{su}(2))$ is unitarily equivalent to a representation $\pi_{N}$ described as follows. The representation space is

$$
\mathcal{M}_{N}:=\bigoplus_{l=|N|,|N|+1, \ldots} \mathcal{V}^{l},
$$

where $\mathcal{V}^{l}$ is a spin l-representation space. The Hilbert space is the closure of $\mathcal{M}_{N}$. The generators of $\mathcal{U}_{q}(\mathrm{su}(2))$ act on each $\mathcal{V}^{l}$ by $\sigma_{l}$ as in (3.4). The representation of the generators $x_{1}, x_{0}, x_{-1}$ of $\mathcal{A}\left(\mathrm{S}_{q t}^{2}\right)$ is determined on an orthonormal basis $\left\{v_{m, N}^{l}: l=|N|,|N|+1, \ldots, m=-l,-l+1, \ldots, l\right\}$ of $\mathcal{M}_{N}$ by

$$
\begin{aligned}
& \pi_{N}\left(x_{i}\right) v_{m, N}^{l} \\
& \quad=\alpha_{i}^{-}(l, m ; N) v_{m+i, N}^{l-1}+\alpha_{i}^{0}(l, m ; N) v_{m+i, N}^{l}+\alpha_{i}^{+}(l, m ; N) v_{m+i, N}^{l+1},
\end{aligned}
$$


where the coefficients are explicitly given by

$$
\begin{aligned}
& \alpha_{1}^{+}(l, m ; N)= q^{-l+m}[l+m+1]^{1 / 2}[l+m+2]^{1 / 2} \\
& \cdot[2 l+1]^{-1 / 2}[2 l+2]^{-1 / 2} \alpha_{N}(l+1), \\
& \alpha_{1}^{0}(l, m ; N)=-q^{m+2}[l-m]^{1 / 2}[l+m+1]^{1 / 2}[2]^{1 / 2}[2 l]^{-1} \beta_{N}(l), \\
& \alpha_{1}^{-}(l, m ; N)=-q^{l+m+1}[l-m-1]^{1 / 2}[l-m]^{1 / 2} \\
& \cdot[2 l-1]^{-1 / 2}[2 l]^{-1 / 2} \alpha_{N}(l), \\
& \alpha_{0}^{+}(l, m ; N)=q^{m}[l-m+1]^{1 / 2}[l+m+1]^{1 / 2}[2]^{1 / 2} \\
& \quad \cdot[2 l+1]^{-1 / 2}[2 l+2]^{-1 / 2} \alpha_{N}(l+1), \\
& \alpha_{0}^{0}(l, m ; N)=[2 l]^{-1}\left([l-m+1][l+m]-q^{2}[l-m][l+m+1]\right) \beta_{N}(l), \\
& \alpha_{0}^{-}(l, m ; N)=q^{m}[l-m]^{1 / 2}[l+m]^{1 / 2}[2]^{1 / 2}[2 l-1]^{-1 / 2}[2 l]^{-1 / 2} \alpha_{N}(l), \\
& \alpha_{-1}^{+}(l, m ; N)=q^{l+m}[l-m+1]^{1 / 2}[l-m+2]^{1 / 2} \\
& \quad \cdot[2 l+1]^{-1 / 2}[2 l+2]^{-1 / 2} \alpha_{N}(l+1), \\
& \alpha_{-1}^{0}(l, m ; N)=q^{m}[l-m+1]^{1 / 2}[l+m]^{1 / 2}[2]^{1 / 2}[2 l]^{-1} \beta_{N}(l), \\
& \alpha_{-1}^{-}(l, m ; N)=-q^{-l+m-1}[l+m-1]^{1 / 2}[l+m]^{1 / 2} \\
& \quad \cdot[2 l-1]^{-1 / 2}[2 l]^{-1 / 2} \alpha_{N}(l)
\end{aligned}
$$

(with the convention that $\alpha_{i}^{0}(0,0 ; 0), \alpha_{i}^{-}(0,0 ; 0), \alpha_{i}^{-}\left(\frac{1}{2}, \pm \frac{1}{2} ; \pm \frac{1}{2}\right)$ are zero). The real numbers $\beta_{N}(l)$ and $\alpha_{N}(l)$ are given by

$$
\begin{aligned}
\alpha_{N}(l)= & \frac{[2]^{1 / 2}[l+N]^{1 / 2}[l-N]^{1 / 2}[2 l]^{1 / 2}}{[2 l+1]^{1 / 2}[l]} \\
& \cdot\left(1-t+q^{-2 N}[2 l]^{-2}[l]^{2}\left(t-1+q^{2 N}\right)^{2}\right)^{1 / 2}, \\
\beta_{N}(l)= & \frac{\epsilon[2|N|]\left(q^{-1}+q-q^{\epsilon} t\right)+t\left(q-q^{-1}\right)\{[|N|][|N|+1]-[l][l+1]\}}{q[2 l+2]},
\end{aligned}
$$

with $\epsilon=\operatorname{sgn}(N)$.

Remark 4.2. In [13], Proposition 6.4, it was shown that the representation space $\mathcal{M}_{N}$ is isomorphic to a projective left $\mathcal{A}\left(\mathrm{S}_{q t}^{2}\right)$-module of rank 1. These projective left $\mathcal{A}\left(\mathrm{S}_{q t}^{2}\right)$-modules can be considered as line bundles over the quantum sphere $\mathrm{S}_{q t}^{2}$ with winding numbers $2 N$ [1], [9], [11]. In particular, $\mathcal{M}_{0} \cong \mathcal{A}\left(\mathrm{S}_{q t}^{2}\right)$ is the trivial line bundle. 
Remark 4.3. The representations $\pi_{ \pm \frac{1}{2}}$ for the standard quantum sphere $\mathcal{A}\left(S_{q 1}^{2}\right)$ appeared first in [7].

4.2. The spin representation. Classically, the spinor bundle on the 2 -sphere is given by the direct sum of two line bundles with winding numbers -1 and 1 . In correspondence with the classical picture, we take

$$
\mathcal{W}:=\mathcal{M}_{-\frac{1}{2}} \oplus \mathcal{M}_{\frac{1}{2}}
$$

as (polynomial sections) of the quantum spinor bundle. The Hilbert space completion $\mathscr{H}$ of $\mathcal{W}$ will be the Hilbert space of spinors with the representation $\pi:=\pi_{-\frac{1}{2}} \oplus \pi_{\frac{1}{2}}$ of the algebra $\mathcal{A}\left(\mathrm{S}_{q t}^{2}\right)$ as the spinor representation.

For simplicity of notation, we shall use the label + instead of $N=\frac{1}{2}$ and instead of $N=-\frac{1}{2}$. With these conventions, $\mathcal{W}:=\mathcal{M}_{-} \oplus \mathcal{M}_{+}$, and $\pi_{ \pm}$denotes the representation $\pi_{ \pm \frac{1}{2}}$. Moreover we shall identify elements of $U_{q}(\operatorname{su}(2))$ with the corresponding operators on $\mathcal{W}$ coming from Proposition 4.1. By that proposition we have the decomposition

$$
\mathcal{W}=\bigoplus_{l=\frac{1}{2}, \frac{3}{2}, \ldots} \mathcal{W}^{l}, \quad \mathcal{W}^{l}=\bigoplus_{m=-l, \ldots, l} \mathcal{W}_{m}^{l},
$$

where $\mathcal{W}_{m}^{l}=\operatorname{span}\left\{v_{m,-}^{l}, v_{m,+}^{l}\right\}$. Throughout this paper, we keep the basis $\left\{v_{m,-}^{l}, v_{m,+}^{l}\right\}$ of $\mathcal{W}_{m}^{l}$ fixed. Clearly, an orthonormal basis of $\mathcal{H}$ is provided by the vectors

$$
\left\{v_{m,-}^{l}, v_{m,+}^{l}: l=\frac{1}{2}, \frac{3}{2}, \ldots, m=-l, \ldots, l\right\} .
$$

For an arbitrary but fixed vector $w_{m}^{l} \in \mathcal{W}_{m}^{l}$, say $w_{m}^{l}=\alpha_{m,-}^{l} v_{m,-}^{l}+\alpha_{m,+}^{l} v_{m,+}^{l}$ with components $\alpha_{m,-}^{l}, \alpha_{m,+}^{l} \in \mathbb{C}$, and for any $i, j \in \mathbb{Z}$, the expression $w_{m+i}^{l+j}$ denotes the vector $w_{m+i}^{l+j}=\alpha_{m,-}^{l} v_{m+i,-}^{l+j}+\alpha_{m,+}^{l} v_{m+i,+}^{l+j} \in \mathcal{W}_{m+i}^{l+j}$. It is understood that $w_{m+i}^{l+j}=0$ whenever $l+j<0$ or $|m+i|>l+j$.

A substantial part of our results will be based on the fact that the operators $\pi\left(x_{-1}\right)$, $\pi\left(x_{0}\right), \pi\left(x_{1}\right)$ can be "approximated" by operators acting diagonally on $\mathcal{M}_{-} \oplus \mathcal{M}_{+}$. This is the content of the next lemma. For this purpose, we define operators $z_{-1}, z_{0}$ and $z_{1}$ on $\mathscr{H}$ by their action on $w_{m}^{l} \in \mathcal{W}_{m}^{l}$ as follows:

$$
z_{i} w_{m}^{l}=\alpha_{i}^{-}(l, m ; 0) w_{m+i}^{l-1}+\alpha_{i}^{0}(l, m ; 0) w_{m+i}^{l}+\alpha_{i}^{+}(l, m ; 0) w_{m+i}^{l+1},
$$

$i=-1,0,1$. Here, the coefficients are the ones defined in equations (4.2) unless $|m+i|>l+v, v=0, \pm 1$; in this case, we set $\alpha_{i}^{v}(l, m ; 0)=0$. Formally, the operators $z_{i}$ 's are given by the same formulae as the $\pi_{0}\left(x_{i}\right)$ 's, but $\mathcal{W}$ and $\mathcal{M}_{0}$ decompose into different spin representation spaces. As a consequence, $z_{-1}, z_{0}$ and 
$z_{1}$ do not satisfy the commutation relations of the generators of $\mathcal{A}\left(\mathrm{S}_{q t}^{2}\right)$. Explicitly, their commutation relations read

$$
\begin{aligned}
z_{-1}\left(z_{0}-t\right) & =q^{2}\left(z_{0}-t\right) z_{-1}, \\
z_{1}\left(z_{0}-t\right) & =q^{-2}\left(z_{0}-t\right) z_{1}, \\
-[2] z_{-1} z_{1}+\left(q^{2} z_{0}+t\right)\left(z_{0}-t\right) & =[2]^{2}(1-t)-c P_{-}, \\
-[2] z_{1} z_{-1}+\left(q^{-2} z_{0}+t\right)\left(z_{0}-t\right) & =[2]^{2}(1-t)-c P_{+},
\end{aligned}
$$

where $P_{ \pm}$denotes the projection onto the 2-dimensional subspace $\operatorname{span}\left\{v_{ \pm 1 / 2,-}^{1 / 2}, v_{ \pm 1 / 2,+}^{1 / 2}\right\}$ and $c:=q[2] \alpha_{0}\left(\frac{1}{2}\right)^{2}=q[2]\left(1-t+\left[\frac{1}{2}\right]^{2} t^{2}\right)$.

Before stating the lemma, we need some more notation. For any bounded operator $L$ on the Hilbert space $\mathscr{H}, \mathcal{l}(L)$ denotes the two-sided ideal of $\mathscr{B}(\mathscr{H})$ generated by $L$. Also, throughout this paper, $L_{q}$ stands for the compact operator on $\mathscr{H}$ defined by

$$
L_{q} w_{m}^{l}=q^{l} w_{m}^{l}, \quad w_{m}^{l} \in \mathcal{W}_{m}^{l} .
$$

Lemma 4.4. There exist bounded operators $A_{i}$ and $B_{i}, i=-1,0,1$, such that

$$
\begin{aligned}
& \pi\left(x_{i}\right)-z_{i}=A_{i} L_{q}^{2}=L_{q}^{2} B_{i} \quad \text { if } t \neq 1, \\
& \pi\left(x_{i}\right)-z_{i}=A_{i} L_{q}=L_{q} B_{i} \quad \text { if } t=1 .
\end{aligned}
$$

In particular, $\pi\left(x_{i}\right)-z_{i} \in \mathcal{l}\left(L_{q}^{2}\right)$ for $t \neq 1$ and $\pi\left(x_{i}\right)-z_{i} \in \mathcal{l}\left(L_{q}\right)$ for $t=1$

Proof. Our aim is to show that $L_{q}^{-k}\left(\pi\left(x_{i}\right)-z_{i}\right)$ and $\left(\pi\left(x_{i}\right)-z_{i}\right) L_{q}^{-k}$ are bounded, with $k=2$ for $t \neq 1$ and $k=1$ for $t=1$. Notice that $\pi\left(x_{i}\right)-z_{i}$ is a sum of three independent weighted shift operators with weights $\alpha_{i}^{v}\left(l, m ; \pm \frac{1}{2}\right)-\alpha_{i}^{v}(l, m ; 0)$, where $v \in\{+,-, 0\}$. Hence it suffices to prove that $q^{-k l}\left|\alpha_{i}^{v}\left(l, m ; \pm \frac{1}{2}\right)-\alpha_{i}^{v}(l, m ; 0)\right| \leq C_{i}^{v}$ for some constants $C_{i}^{v} \in \mathbb{R}^{+}$. Using the inequalities

$$
[n] \leq\left(q^{-1}-q\right)^{-1} q^{-n} \text { for all } n \geq 0, \quad[n]^{-1} \leq q^{n-1} \text { for all } n \geq 1,
$$

one verifies that the coefficients in front of $\alpha_{N}(l)$ and $\beta_{N}(l)$ in equations (4.2a)-(4.2c) are uniformly bounded. Thus, we only have to prove that the sequences $q^{-k l}\left|\beta_{N}(l)-\beta_{0}(l)\right|$ and $q^{-k l}\left|\alpha_{N}(l)-\alpha_{0}(l)\right|$ are bounded. We prove this for $N \in \frac{1}{2} \mathbb{Z}$, even though we need it only for $N= \pm 1 / 2$. The boundedness of the first sequence follows from the identity

$$
\left|\beta_{N}(l)-\beta_{0}(l)\right|=\frac{\left|\epsilon[2|N|]\left(q^{-1}+q-q^{\epsilon} t\right)+t\left(q-q^{-1}\right)[|N|][|N|+1]\right|}{q[2 l+2]},
$$

using $(q[2 l+2])^{-1} \leq q^{2 l}$. Concerning the second sequence, if $t=1$ one verifies directly that for any $N$ the sequence $q^{-l}\left|\alpha_{N}(l)\right|$ is bounded; thus $q^{-l}\left|\alpha_{N}(l)-\alpha_{0}(l)\right|$ 
is bounded. If $t \in[0,1)$, set

$$
u_{l}:=\frac{[l+N][l-N][2 l]}{q[2 l+1][l]^{2}} \frac{1-t+q^{-2 N}[2 l]^{-2}[l]^{2}\left(t-1+q^{2 N}\right)^{2}}{1-t}-1
$$

for $l \geq 1$. From $[l+N][l-N][2 l] \leq q[2 l+1][l]^{2}$ we obtain

$$
u_{l} \leq \frac{q^{-2 N}[2 l]^{-2}[l]^{2}\left(t-1+q^{2 N}\right)^{2}}{1-t}=C_{N}\left(1-q^{2}\right)^{2}[2 l]^{-2}[l]^{2} \leq C_{N} q^{2 l},
$$

with $C_{N}:=\frac{\left\{q^{N}-q^{-N}(1-t)\right\}^{2}}{\left(1-q^{2}\right)^{2}(1-t)}$. On the other hand from $[l+N][l-N]=[l]^{2}-[N]^{2}$ and $[2 l]-q[2 l+1]=-q^{2 l+1}$ we get

$u_{l} \geq \frac{[2 l][l+N][l-N]}{q[2 l+1][l]^{2}}-1=\frac{-[2 l][N]^{2}-q^{2 l+1}[l]^{2}}{q[2 l+1][l]^{2}} \geq-q^{2 l-2} \frac{[N]^{2}}{1-q^{2}}-q^{4 l-1}$.

Hence there exists a constant $C_{N}^{\prime} \in \mathbb{R}^{+}$such that $\left|u_{l}\right| \leq C_{N}^{\prime} q^{2 l}$. Moreover from the inequality $|\sqrt{1+u}-1| \leq|u|$ which holds for any $u \geq-1$, it follows that

$$
\left|\frac{\alpha_{N}(l)}{\sqrt{\left(1+q^{2}\right)(1-t)}}-1\right|=\left|\sqrt{1+u_{l}}-1\right| \leq\left|u_{l}\right| \leq C_{N}^{\prime} q^{2 l} .
$$

Finally, using the triangle inequality we conclude that $\frac{\left|\alpha_{N}(l)-\alpha_{0}(l)\right|}{\sqrt{\left(1+q^{2}\right)(1-t)}} \leq\left(C_{0}+C_{N}\right) q^{2 l}$.

\section{Equivariant Dirac operators}

In this section, we provide a class of equivariant self-adjoint operators $D$ on the Hilbert space of spinors $\mathscr{H}$ such that $\left(\mathcal{A}\left(\mathrm{S}_{q t}^{2}\right), \mathscr{H}, D\right)$ fulfills all conditions of a spectral triple.

Let $C_{q}:=q k^{2}+q^{-1} k^{-2}+\left(q-q^{-1}\right)$ ef be the Casimir operator of $\mathcal{U}_{q}(\mathrm{su}(2))$. It acts on the space of spinors $\mathcal{W}$ by $C_{q} v_{m, \pm}^{l}=\left(q^{2 l+1}+q^{-2 l-1}\right) v_{m, \pm}^{l}$, and its closure on $\mathscr{H}$ is self-adjoint. Suppose that $D$ is a self-adjoint operator on $\mathscr{H}$ commuting strongly with the closure of $C_{q}$. Then the finite-dimensional subspaces $\mathbb{W}^{l}$ reduce $D$, and $\mathcal{W}$ is invariant under $D$. Assume next that $D$ is equivariant, i.e., $X D=D X$ for all $X \in \mathcal{U}_{q}(\mathrm{su}(2))$.

From $k D=D k$, it follows that $D$ leaves the 2-dimensional subspaces $\mathfrak{W}_{m}^{l}$ invariant. Let $D_{m}^{l}$ be a self-adjoint operator on $\mathbb{C}^{2}$ such that the restriction of $D$ to $W_{m}^{l}$, in our fixed basis for the latter, is given by $D_{m}^{l}$. Then $D f=f D$ implies that $D_{m+1}^{l}=D_{m}^{l}$. Hence there exists a self-adjoint operator on $\mathbb{C}^{2}, D_{l}$ say, such that $D_{m}^{l}=D_{l}$ for all $m=-l, \ldots, l$. Diagonalizing $D_{l}$, we can write

$$
D_{l}=U_{l}^{*}\left(\begin{array}{cc}
d_{l}^{\uparrow} & 0 \\
0 & d_{l}^{\downarrow}
\end{array}\right) U_{l},
$$


where $d_{l}^{\uparrow}$ and $d_{l}^{\downarrow}$ are the eigenvalues of $D_{l}$ and $U_{l}$ is a unitary operator on $\mathbb{C}^{2}$. Without loss of generality, we may assume that $d_{l}^{\downarrow} \leq d_{l}^{\uparrow}$. Definition 2.1 (i) is fulfilled if and only if

$$
\lim _{l \rightarrow \infty}\left|d_{l}^{\uparrow}\right|=\lim _{l \rightarrow \infty}\left|d_{l}^{\downarrow}\right|=\infty .
$$

Next we give sufficient conditions for the boundedness of the commutators $[D, \pi(a)]$ with $a \in \mathcal{A}\left(\mathrm{S}_{q t}^{2}\right)$. By the Leibniz rule for the commutator, $[D, x y]=$ $[D, x] y+x[D, y]$, it suffices to consider only commutators with the generators $x_{-1}$, $x_{0}, x_{1}$. As the action of the operators $z_{-1}, z_{0}, z_{1}$ from Lemma 4.4 is comparatively simple, we write $\left[D, \pi\left(x_{i}\right)\right]=\left[D, z_{i}\right]+\left[D, \pi\left(x_{i}\right)-z_{i}\right]$ and start by analyzing $\left[D, z_{i}\right]$. Let $w_{m}^{l} \in \mathcal{W}_{m}^{l}$. Then

$$
\begin{aligned}
{\left[D, z_{i}\right] w_{m}^{l}=} & \alpha_{i}^{-}(l, m ; 0)\left(U_{l-1}^{*}\left(\begin{array}{cc}
d_{l-1}^{\uparrow} & 0 \\
0 & d_{l-1}^{\downarrow}
\end{array}\right) U_{l-1}-U_{l}^{*}\left(\begin{array}{cc}
d_{l}^{\uparrow} & 0 \\
0 & d_{l}^{\downarrow}
\end{array}\right) U_{l}\right) w_{m+i}^{l-1} \\
& +\alpha_{i}^{+}(l, m ; 0)\left(U_{l+1}^{*}\left(\begin{array}{cc}
d_{l+1}^{\uparrow} & 0 \\
0 & d_{l+1}^{\downarrow}
\end{array}\right) U_{l+1}-U_{l}^{*}\left(\begin{array}{cc}
d_{l}^{\uparrow} & 0 \\
0 & d_{l}^{\downarrow}
\end{array}\right) U_{l}\right) w_{m+i}^{l+1} .
\end{aligned}
$$

We need to treat the cases $t \neq 1$ and $t=1$ separately.

Firstly, let $t \neq 1$. Using (4.7) and observing that

$$
\lim _{l \rightarrow \infty} \alpha_{ \pm \frac{1}{2}}(l)=\left(1+q^{2}\right)^{1 / 2}(1-t)^{1 / 2}
$$

we deduce that the sequence $\left\{\alpha_{i}^{ \pm}(l, m ; 0)\right\}$ is uniformly bounded and does not converge to zero. Hence $\left[D, z_{i}\right]$ is bounded if and only if there exist $C \in \mathbb{R}$ such that

$$
\left\|U_{l+1}^{*}\left(\begin{array}{cc}
d_{l+1}^{\uparrow} & 0 \\
0 & d_{l+1}^{\downarrow}
\end{array}\right) U_{l+1}-U_{l}^{*}\left(\begin{array}{cc}
d_{l}^{\uparrow} & 0 \\
0 & d_{l}^{\downarrow}
\end{array}\right) U_{l}\right\|<C \quad \text { for all } l=\frac{1}{2}, \frac{2}{3}, \ldots,
$$

or, equivalently,

$$
\left\|U_{l} U_{l+1}^{*}\left(\begin{array}{cc}
d_{l+1}^{\uparrow} & 0 \\
0 & d_{l+1}^{\downarrow}
\end{array}\right) U_{l+1} U_{l}^{*}-\left(\begin{array}{cc}
d_{l}^{\uparrow} & 0 \\
0 & d_{l}^{\downarrow}
\end{array}\right)\right\|<C \quad \text { for all } l=\frac{1}{2}, \frac{2}{3}, \ldots
$$

Denoting by $t_{l}$ the absolute value of the $(1,1)$ entry of the matrix $U_{l} U_{l+1}^{*}$ the last inequality implies that there is a fixed constant $C^{\prime} \in \mathbb{R}$ satisfying

$$
\begin{array}{r}
\left|d_{l+1}^{\uparrow}-d_{l}^{\uparrow}-t_{l}^{2}\left(d_{l+1}^{\uparrow}-d_{l+1}^{\downarrow}\right)\right|=\left|d_{l+1}^{\downarrow}-d_{l}^{\uparrow}+\left(1-t_{l}^{2}\right)\left(d_{l+1}^{\uparrow}-d_{l+1}^{\downarrow}\right)\right|<C^{\prime}, \\
\left|d_{l+1}^{\downarrow}-d_{l}^{\downarrow}+t_{l}^{2}\left(d_{l+1}^{\uparrow}-d_{l+1}^{\downarrow}\right)\right|=\left|d_{l+1}^{\uparrow}-d_{l}^{\downarrow}-\left(1-t_{l}^{2}\right)\left(d_{l+1}^{\uparrow}-d_{l+1}^{\downarrow}\right)\right|<C^{\prime}, \\
t_{l} \sqrt{1-t_{l}^{2}}\left|d_{l+1}^{\uparrow}-d_{l+1}^{\downarrow}\right|<C^{\prime},
\end{array}
$$


for all $l=\frac{1}{2}, \frac{2}{3}, \ldots$ Using $t_{l} \in[0,1]$ and $d_{l+1}^{\uparrow} \geq d_{l+1}^{\downarrow}$, one deduces that

$$
\sup \left\{\left|d_{l+1}^{\downarrow}-d_{l}^{\downarrow}\right|,\left|d_{l+1}^{\uparrow}-d_{l}^{\uparrow}\right|: l=\frac{1}{2}, \frac{2}{3}, \ldots\right\}<\infty .
$$

Consequently, there exist $C \in \mathbb{R}$ such that

$$
\left|d_{l}^{\downarrow}\right|<C l, \quad\left|d_{l}^{\uparrow}\right|<C l .
$$

From these inequalities, it follows that both $D L_{q}^{2}$ and $L_{q}^{2} D$ are bounded and so is $\left[D, \pi\left(x_{i}\right)-z_{i}\right]$ by Lemma 4.4. Hence equation (5.4) implies that both commutators $\left[D, z_{i}\right]$ and $\left[D, \pi\left(x_{i}\right)-z_{i}\right]$ are bounded and so is their sum $\left[D, \pi\left(x_{i}\right)\right]$.

If $t=1$, a direct computation shows that the sequence $\left\{q^{-l} \alpha_{i}^{ \pm}(l, m ; 0)\right\}$ is uniformly bounded and does not converge to zero. As a consequence, $\left[D, z_{i}\right]$ is bounded if and only if there are $C \in \mathbb{R}$ such that

$$
\left\|U_{l+1}^{*}\left(\begin{array}{cc}
d_{l+1}^{\uparrow} & 0 \\
0 & d_{l+1}^{\downarrow}
\end{array}\right) U_{l+1}-U_{l}^{*}\left(\begin{array}{cc}
d_{l}^{\uparrow} & 0 \\
0 & d_{l}^{\downarrow}
\end{array}\right) U_{l}\right\|<C q^{-l} \text { for all } l=\frac{1}{2}, \frac{2}{3}, \ldots
$$

On the other hand, a sufficient condition for the commutator $\left[D, \pi\left(x_{i}\right)-z_{i}\right]$ to be bounded is that $D L_{q}=L_{q} D$ is bounded (since by Lemma 4.4, $\pi\left(x_{i}\right)-z_{i}=A_{i} L_{q}=$ $L_{q} B_{i}$ with bounded operators $A_{i}$ and $B_{i}$ ). Now the operator $D L_{q}=L_{q} D$ is bounded if and only if there exist $C \in \mathbb{C}$ such that

$$
\left|d_{l}^{\downarrow}\right|<C q^{-l}, \quad\left|d_{l}^{\uparrow}\right|<C q^{-l}, \quad l=\frac{1}{2}, \frac{2}{3}, \ldots
$$

Since these inequalities imply (5.7), they are sufficient for the commutator $\left[D, \pi\left(x_{i}\right)\right]=\left[D, z_{i}\right]+\left[D, \pi\left(x_{i}\right)-z_{i}\right]$ to be bounded.

Summarizing, we have established the following proposition.

Proposition 5.1. Let $D$ be an equivariant self-adjoint operator on $\mathscr{H}$ such that $D$ and the closure of $C_{q}$ strongly commute. Then $\mathcal{W}_{m}^{l}=\operatorname{span}\left\{v_{m,-}^{l}, v_{m,+}^{l}\right\}$ is an invariant subspace and the restriction of $D$ to $\mathrm{W}_{m}^{l}$ is given by (5.1). The triple $\left(\mathcal{A}\left(\mathrm{S}_{q t}^{2}\right), \mathcal{H}, D\right)$ defines an $U_{q}(\operatorname{su}(2))$ equivariant spectral triple if, for $t \neq 1$, conditions (5.2) and (5.4) are satisfied; and for $t=1$, conditions (5.2) and (5.8) are satisfied.

\section{The real structure}

We require that the real structure $J$ be equivariant. This means that $J$ is the antiunitary part of a closed antilinear operator $T$ satisfying $T X=S(X)^{*} T$ for all $X \in \mathcal{U}_{q}(\operatorname{su}(2))$ (cf. Section 2). Now, any antiunitary operator $J$ which leaves $\mathcal{W}$ invariant and fulfills

$$
J X J^{-1}=k S(X)^{*} k^{-1}, \quad X \in \mathcal{U}_{q}(\operatorname{su}(2)),
$$


is equivariant. To see this, one can take for $T$ the closure of the operator $J k$ since $J k X=S(X)^{*} J k$ for all $X \in U_{q}(\operatorname{su}(2))$.

Next, consider the antiunitary operator $J_{0}$ on $\mathscr{H}$ defined by

$$
J_{0} v_{m, \pm}^{l}=(-1)^{m+1 / 2} v_{-m, \pm}^{l}, \quad l=\frac{1}{2}, \frac{2}{3}, \ldots, m=-l, \ldots, l .
$$

Clearly, $J_{0}^{2}=-1$. The antiunitary operator $J_{0}$ will play a crucial role in discussing the general form of an equivariant real structure. We summarize some properties of $J_{0}$ in the following lemmata.

Lemma 6.1. The antiunitary operator $J_{0}$ is equivariant.

Proof. First note that $J_{0}$ leaves the spinor bundle $W$ invariant. The lemma is proved by showing that $J_{0}$ satisfies (6.1). Since $X \mapsto k S(X)^{*} k^{-1}$ is an antilinear homomorphism, it suffices to verify (6.1) for the generators of $U_{q}(\operatorname{su}(2))$ which can easily be done by straightforward calculations.

Lemma 6.2. With the operators $z_{-1}, z_{0}, z_{1}$ defined in equation (4.5),

$$
\left[z_{i}, J_{0} z_{j} J_{0}^{-1}\right]=0, \quad i, j=-1,0,1 .
$$

Proof. In the notation of equation (4.5), we have

$$
\begin{aligned}
& J_{0} z_{i} J_{0}^{-1} w_{m}^{l} \\
& =(-1)^{i}\left(\alpha_{i}^{-}(l,-m ; 0) w_{m-i}^{l-1}+\alpha_{i}^{0}(l,-m ; 0) w_{m-i}^{l}+\alpha_{i}^{+}(l,-m ; 0) w_{m-i}^{l+1}\right)
\end{aligned}
$$

for $w_{m}^{l} \in \mathcal{W}_{m}^{l}$. The lemma is proved by direct computations using equations (4.5) and (6.4).

Lemma 6.3. Let $d$ denotes the operator ideal $d\left(L_{q}\right)$ or $d\left(L_{q}^{2}\right)$ for $t=1$ and $t \neq 1$, respectively. Then, for all $a, b \in \mathcal{A}\left(\mathrm{S}_{q t}^{2}\right)$,

$$
\left[\pi(a), J_{0} \pi(b) J_{0}^{-1}\right] \in \mathcal{\ell} .
$$

Proof. Again from the Leibniz rule of commutators, it suffices to prove equation (6.5) for the generators $x_{-1}, x_{0}, x_{1}$. Since

$$
\begin{aligned}
{\left[\pi\left(x_{i}\right), J_{0} \pi\left(x_{j}\right) J_{0}^{-1}\right]=} & {\left[\pi\left(x_{i}\right)-z_{i}, J_{0} \pi\left(x_{j}\right) J_{0}^{-1}\right] } \\
& +\left[z_{i}, J_{0}\left(\pi\left(x_{j}\right)-z_{j}\right) J_{0}^{-1}\right]+\left[z_{i}, J_{0} z_{j} J_{0}^{-1}\right],
\end{aligned}
$$

the assertion follows from Lemmata 4.4 and 6.2. 
Lemma 6.4. Let $\left(\mathcal{A}\left(\mathrm{S}_{q t}^{2}\right), \mathcal{H}, D, J\right)$ be an equivariant 2-dimensional real spectral triple. Assume that $J$ satisfies (6.1). Then there exist unitary operators $W_{l}$ on $\mathbb{C}^{2}$ such that the restrictions of $D$ and $J$ to $\mathrm{W}_{m}^{l}$ can be expressed as

$$
\begin{aligned}
& D\left\lceil_{w_{m}^{l}}=D_{l}=W_{l}^{*}\left(\begin{array}{cc}
d_{l}^{\uparrow} & 0 \\
0 & d_{l}^{\downarrow}
\end{array}\right) W_{l},\right. \\
& J\left\lceil_{w_{m}^{l}}=W_{l}^{*} \bar{W}_{l} J_{0}\left\lceil_{w_{m}^{l}}=W_{l}^{*} J_{0}\left\lceil_{w_{m}^{l}} W_{l},\right.\right.\right.
\end{aligned}
$$

where the bar denotes complex conjugation.

Proof. Given an antiunitary operator $J$ satisfying (6.1), the operator $V=J J_{0}^{-1}=$ $-J J_{0}$ is unitary and fulfills for all $X \in U_{q}(\operatorname{su}(2))$

$$
V X V^{*}=J J_{0} X J_{0}^{-1} J^{-1}=S\left(S(X)^{*}\right)^{*}=X
$$

since, for any Hopf $*$-algebra, $* \circ S \circ * \circ S=$ id. In particular, $V$ commutes with $k$ and with the Casimir operator $C_{q}$ and does so with their closures. By an argument similar to the one at the beginning of Section 5, one shows that there exist unitary operators $V_{l}$ on $\mathbb{C}^{2}$ such that the restriction of $V$ to $\mathcal{W}_{m}^{l}$ is given by $V_{l}$. Thus

$$
J=V J_{0}, \quad J\left\lceil_{w_{m}^{l}}=V_{l} J_{0} \Gamma_{w_{m}^{l}} .\right.
$$

Recall that, in our standard basis, $D_{l}=D\left\lceil_{w_{m}^{l}}\right.$ has the form (5.1). As $J_{0}$ is antiunitary, we get

$$
J_{0}\left\lceil_{w_{m}^{l}} D_{l}=\bar{D}_{l} J_{0}\left\lceil_{w_{m}^{l}}\right.\right.
$$

(the bar is still complex conjugation). From the requirement $J D J^{-1}=D$, it follows that $D_{l}=V_{l} \bar{D}_{l} V_{l}^{*}$ or, equivalently,

$$
\left(\begin{array}{cc}
d_{l}^{\uparrow} & 0 \\
0 & d_{l}^{\downarrow}
\end{array}\right)=U_{l} V_{l} \bar{U}_{l}^{*}\left(\begin{array}{cc}
d_{l}^{\uparrow} & 0 \\
0 & d_{l}^{\downarrow}
\end{array}\right)\left(U_{l} V_{l} \bar{U}_{l}^{*}\right)^{*}
$$

If $d_{l}^{\uparrow} \neq d_{l}^{\downarrow}$, the last equation implies that $U_{l} V_{l} \bar{U}_{l}^{*}$ is a diagonal matrix. Thus there are angle variables $\phi_{l}, \psi_{l} \in[0,2 \pi)$ such that

$$
V_{l}=U_{l}^{*}\left(\begin{array}{cc}
\exp \left(\mathrm{i} \phi_{l}\right) & 0 \\
0 & \exp \left(\mathrm{i} \psi_{l}\right)
\end{array}\right) \bar{U}_{l} .
$$

Inserting

$$
W_{l}:=\left(\begin{array}{cc}
\exp \left(-\frac{\mathrm{i}}{2} \phi_{l}\right) & 0 \\
0 & \exp \left(-\frac{\mathrm{i}}{2} \psi_{l}\right)
\end{array}\right) U_{l}
$$

into (5.1) and (6.8), we arrive at (6.6) and (6.7). Clearly, equation (6.6) remains valid if $d_{l}^{\uparrow}=d_{l}^{\downarrow}$ and $W_{l}$ is an arbitrary unitary matrix. Therefore we may assume without loss of generality that $D$ and $J$ are given on $W$ by equations (6.6) and (6.7), respectively. 
Under the assumption that $J$ satisfies (6.1), Lemma 6.4 provides necessary conditions for equivariant real spectral triples. Next, we aim at finding sufficient conditions for $(\mathcal{A}, \mathscr{H}, D, J)$ to yield an equivariant real spectral triple up to infinitesimals. The ideal $d$ from Definition 2.2 is generated by operators $L_{q}^{\beta}$, where $\beta$ is a positive real constant. Again, the basic idea of the proof is to replace the generators $\pi\left(x_{i}\right)$ by the simpler operators $z_{i}$. By the last lemma, the operator $D$ and $J$ act diagonally with respect to the basis formed by $\tilde{v}_{m, \pm}^{l}:=W_{l}^{*} v_{m, \pm}^{l}$, but the operators $z_{i}$ do not do so (in general). Their action on this basis is found to be

$$
\begin{aligned}
z_{i} \tilde{v}_{m, \pm}^{l}= & \alpha_{i}^{-}(l, m ; 0) W_{l} W_{l-1}^{*} \tilde{v}_{m+i, \pm}^{l-1} \\
& +\alpha_{i}^{0}(l, m ; 0) \tilde{v}_{m+i, \pm}^{l}+\alpha_{i}^{+}(l, m ; 0) W_{l} W_{l+1}^{*} \tilde{v}_{m+i, \pm}^{l+1}
\end{aligned}
$$

In order to reduce the computations to diagonal operators, we require thus that

$$
\left\|W_{l} W_{l+1}^{*}-1\right\|<C q^{\beta l}
$$

for some real constant $C$. Then (6.9) implies that, modulo the ideal $d\left(L_{q}^{\beta}\right)$, the operators $z_{i}$ act on the basis vectors $\tilde{v}_{m, \pm}^{l}$ as a sum of diagonal shift operators. Next, with the operators $z_{i}$ acting on the basis vectors $\tilde{v}_{m, \pm}^{l}$ as diagonal shifts, we compute

$$
\begin{aligned}
& \quad\left|\left\langle\tilde{v}_{m+i-j,+}^{l+2},\left[\left[D, z_{i}\right], J z_{j} J^{-1}\right] \tilde{v}_{m,+}^{l}\right\rangle\right| \\
& \quad=\alpha_{i}^{+}(l+1, m-j ; 0) \alpha_{j}^{+}(l,-m ; 0)\left|\left(d_{l+2}^{\uparrow}-d_{l+1}^{\uparrow}\right)-\left(d_{l+1}^{\uparrow}-d_{l}^{\uparrow}\right)\right|,
\end{aligned}
$$

where we used $\alpha_{i}^{+}(l+1, m-j ; 0) \alpha_{j}^{+}(l,-m ; 0)=\alpha_{j}^{+}(l+1,-m-i ; 0) \alpha_{i}^{+}(l, m ; 0)$.

Let $t \neq 1$. To ensure that $\left|\left\langle\tilde{v}_{m+i-j,+}^{l+2},\left[\left[D, z_{i}\right], J z_{j} J^{-1}\right] \tilde{v}_{m,+}^{l}\right\rangle\right|<C q^{\beta l}$ for some real constant $C$, we must impose the condition

$$
\left|\left(d_{l+2}^{\uparrow}-d_{l+1}^{\uparrow}\right)-\left(d_{l+1}^{\uparrow}-d_{l}^{\uparrow}\right)\right|<C q^{\beta l},
$$

since $\alpha_{i}^{+}(l+1, m-j ; 0) \alpha_{j}^{+}(l,-m ; 0)=O(1)$. A similar argument leads also to

$$
\left|\left(d_{l+2}^{\downarrow}-d_{l+1}^{\downarrow}\right)-\left(d_{l+1}^{\downarrow}-d_{l}^{\downarrow}\right)\right|<C q^{\beta l} .
$$

From equation (2.3), Lemma 6.4 and the foregoing, it is immediately clear that one can always add to $D$ a self-adjoint operator from $\ell\left(L_{q}^{\beta}\right)$ having the same eigenvectors. A sufficient condition for equations (6.10) and (6.11) to be satisfied is to assume that the eigenvalues $d_{l}^{\uparrow}$ and $d_{l}^{\downarrow}$ depend linearly on $l$. But this dependence is alike the one in (5.6) to get bounded commutators of $D$ with algebra elements.

On the other hand, for $t=1$, the argument leading to equations (6.10) and (6.11) fails since in this case $\alpha_{i}^{+}(l, m ; 0)=O\left(q^{l}\right)$. However, a linear dependence on the eigenvalues of $D$ clearly satisfies the condition (5.8) for bounded commutators in 
this case, too. Thus, by Proposition 5.1, for all Podleś quantum spheres, we have equivariant spectral triples $\left(\mathcal{A}\left(\mathrm{S}_{q t}^{2}\right), \mathscr{H}, D\right)$ when the eigenvalues $d_{l}^{\uparrow}$ and $d_{l}^{\downarrow}$ of $D$ depend linearly on $l$.

The next proposition shows that this linear dependence, together with the requirement (6.9), suffices to obtain equivariant real spectral triples (up to infinitesimals) for all quantum spheres $\mathcal{A}\left(\mathrm{S}_{q t}^{2}\right)$.

Proposition 6.5. Suppose that

$$
d_{l}^{\uparrow}=c_{1}^{\uparrow} l+c_{2}^{\uparrow}, \quad d_{l}^{\downarrow}=c_{1}^{\downarrow} l+c_{2}^{\downarrow},
$$

where $c_{1}^{\uparrow}, c_{2}^{\uparrow}, c_{1}^{\downarrow}, c_{2}^{\downarrow}$ are real numbers such that $c_{1}^{\uparrow} \neq 0$ and $c_{1}^{\downarrow} \neq 0$. Let $D$ be the self-adjoint operator on $\mathscr{H}$ determined by (6.6), and let the antiunitary operator $J$ be given by (6.7). Suppose that $\beta \in(0,2]$ for $t \neq 1$ and $\beta \in(0,1]$ for $t=1$, and assume that (6.9) holds. Then $\left(\mathcal{A}\left(\mathrm{S}_{\text {qt }}^{2}\right), \mathcal{H}, D, J\right)$ defines an equivariant real spectral triple such that equation (2.3) is satisfied with $\mathcal{l}:=d\left(L_{q}^{\alpha}\right)$, where $\alpha \in(0, \beta)$.

Proof. Clearly, equations (6.6) and (6.12) uniquely determine a self-adjoint operator (denoted by $D$ ) on $\mathscr{H}$ since the collection $\left\{W_{l}^{*} v_{m,-}^{l}, W_{l}^{*} v_{m,+}^{l}: l=\frac{1}{2}, \frac{2}{3}, \ldots, m=\right.$ $-l, \ldots, l\}$ is a complete set of orthonormal eigenvectors. By Lemma 6.4 , it is evident that $J D=D J$ and that $J^{2}=-1$. Lemma 6.1 and equation (6.7) imply that (6.1) also applies, so $J$ is equivariant. Thus it remains to verify equation (2.3).

Observe that $\ell\left(L_{q}^{\alpha_{1}}\right) \subset \mathcal{l}\left(L_{q}^{\alpha_{2}}\right)$ for $\alpha_{2} \geq \alpha_{1}>0$. From

$$
\begin{aligned}
\left(J z_{i} J^{-1}-J_{0} z_{i} J_{0}^{-1}\right) w_{m}^{l}=(-1)^{i} & \left(\alpha_{i}^{-}(l,-m ; 0)\left(W_{l-1}^{*} \bar{W}_{l-1} \bar{W}_{l}^{*} W_{l}-1\right) w_{m-i}^{l-1}\right. \\
& \left.+\alpha_{i}^{+}(l,-m ; 0)\left(W_{l+1}^{*} \bar{W}_{l+1} \bar{W}_{l}^{*} W_{l}-1\right) w_{m-i}^{l+1}\right)
\end{aligned}
$$

and equation (6.9), it follows that $J z_{i} J^{-1}-J_{0} z_{i} J_{0}^{-1} \in \mathcal{l}\left(L_{q}^{\beta}\right)$. As a consequence, and using Lemma 6.2, one finds that

$$
\left[z_{i}, J z_{j} J^{-1}\right]=\left[z_{i}, J_{0} z_{j} J_{0}^{-1}\right]+\left[z_{i}, J z_{i} J^{-1}-J_{0} z_{j} J_{0}^{-1}\right] \in \mathcal{l}\left(L_{q}^{\beta}\right) .
$$

Now the proof of the first equation of (2.3) is completely analogous to the proof of Lemma 6.3 with $J_{0}$ replaced by $J$.

By a repeated use of the Leibniz rule for the commutator and of the first relation of (2.3), we need to prove the second relation of (2.3) only for the generators $x_{-1}, x_{0}$, $x_{1}$. Note that the operator $D L_{q}^{\beta-\alpha}=L_{q}^{\beta-\alpha} D$ is bounded. Hence, by Lemma 4.4, it follows that $\left[D, \pi\left(x_{i}\right)-z_{i}\right] \in \mathcal{l}\left(L_{q}^{\alpha}\right)$. Now, writing

$$
\begin{aligned}
{\left[\left[D, \pi\left(x_{i}\right)\right], J \pi\left(x_{j}\right) J^{-1}\right]=} & {\left[\left[D, \pi\left(x_{i}\right)-z_{i}\right], J \pi\left(x_{j}\right) J^{-1}\right] } \\
& +\left[\left[D, z_{i}\right], J\left(\pi\left(x_{j}\right)-z_{j}\right) J^{-1}\right]+\left[\left[D, z_{i}\right], J z_{j} J^{-1}\right]
\end{aligned}
$$


and using again Lemma 4.4, we see that it suffices to establish that

$$
\left[\left[D, z_{i}\right], J z_{j} J^{-1}\right] \in \mathcal{l}\left(L_{q}^{\alpha}\right) .
$$

Let $W$ denote the unitary operator on $\mathscr{H}$ given by $W w_{m}^{l}=W_{l} w_{m}^{l}$ for all $w_{m}^{l} \in W_{m}^{l}$. Then $J=W^{*} J_{0} W$ and $D=W^{*} \widetilde{D} W$, where $\widetilde{D}$ is the unique self-adjoint operator on $\mathscr{H}$ such that

$$
\widetilde{D}\left\lceil_{w_{m}^{l}}=\widetilde{D}_{l}:=\left(\begin{array}{cc}
d_{l}^{\uparrow} & 0 \\
0 & d_{l}^{\downarrow}
\end{array}\right) .\right.
$$

In these terms, the requirement (6.14) is equivalent to

$$
\left[\left[\widetilde{D}, W z_{i} W^{*}\right], J_{0} W z_{j} W^{*} J_{0}^{-1}\right] \in \mathcal{d}\left(L_{q}^{\alpha}\right) .
$$

Evaluating $W z_{i} W^{*}-z_{i}$ on vectors $w_{m}^{l} \in W_{m}^{l}$ gives

$\left(W z_{i} W^{*}-z_{i}\right) w_{m}^{l}=\alpha_{i}^{-}(l, m)_{0}\left(W_{l-1} W_{l}^{*}-1\right) w_{m+i}^{l-1}+\alpha_{i}^{+}(l, m)_{0}\left(W_{l+1} W_{l}^{*}-1\right) w_{m+i}^{l+1}$.

From this and (6.9), we conclude that $W z_{i} W^{*}-z_{i} \in \ell\left(L_{q}^{\beta}\right) \subset d\left(L_{q}^{\alpha}\right)$. Thus, equation (6.16) is, in turn, equivalent to

$$
\left[\left[\widetilde{D}, z_{i}\right], J_{0} z_{j} J_{0}^{-1}\right] \in \mathcal{l}\left(L_{q}^{\alpha}\right) .
$$

Note now that $\left[\left[\widetilde{D}, z_{i}\right], J_{0} z_{j} J_{0}^{-1}\right]$ can be written as a sum of five independent weighted shift operators with weights $S_{i, j}^{v}(l, m), v=-2, \ldots, 2$, i.e.,

$$
\left[\left[\widetilde{D}, z_{i}\right], J_{0} z_{j} J_{0}^{-1}\right] w_{m}^{l}=\sum_{\nu=-2}^{2} S_{i, j}^{v}(l, m) w_{m+i-j}^{l+v}, \quad w_{m}^{l} \in \mathcal{W}_{m}^{l} .
$$

Moreover,

$$
\left[\widetilde{D}, z_{i}\right] v_{m, \pm}^{l}=c_{1}^{ \pm}\left(\alpha_{i}^{+}(l, m ; 0) v_{m+i, \pm}^{l+1}-\alpha_{i}^{-}(l, m ; 0) v_{m+i, \pm}^{l-1}\right),
$$

where $c_{1}^{+}=c_{1}^{\uparrow}$ and $c_{1}^{-}=c_{1}^{\downarrow}$. Using (6.19) and (6.4), a lengthy but straightforward computations shows that $\left|S_{i, j, \pm}^{v}(l, m)\right|<C q^{2 l}$ for some $C \in \mathbb{R}$. From this, we conclude that $\left[\left[\tilde{D}, z_{i}\right], J_{0} z_{j} J_{0}^{-1}\right] \in \mathcal{l}\left(L_{q}^{2}\right)$ which implies (6.17), and the proof is complete.

Corollary 6.6. Up to adding elements from the ideal $d\left(L_{q}^{\alpha}\right)$, the operators $D$ and $J$ from Proposition 6.5 are given by

$$
\begin{aligned}
& D\left\lceil_{w_{m}^{l}}=W_{0}^{*}\left(\begin{array}{cc}
d_{l}^{\uparrow} & 0 \\
0 & d_{l}^{\downarrow}
\end{array}\right) W_{0},\right. \\
& J\left\lceil_{w_{m}^{l}}=W_{0}^{*} J_{0}\left\lceil_{w_{m}^{l}} W_{0},\right.\right.
\end{aligned}
$$

where $W_{0}$ is a unitary operator on $\mathbb{C}^{2}$. 
Proof. Let $D_{1}$ and $J_{1}$ be given by equations (6.6) and (6.7), and suppose that the unitary operators $W_{l}$ satisfy (6.9). Then $\left\{W_{l}\right\}_{l=\frac{1}{2}, \frac{2}{3}, \ldots}$ is a Cauchy sequence and $W_{0}:=\lim _{l \rightarrow \infty} W_{l}$ is a unitary operator. Let $D$ and $J$ denote the operators determined by (6.20) and (6.21). With $\widetilde{D}_{l}$ the diagonal matrix defined in (6.15), we have

$$
\left(D_{1}-D\right) w_{m}^{l}=\left(W_{l} \widetilde{D}_{l} W_{l}-W_{0} \widetilde{D}_{l} W_{0}\right) w_{m}^{l},
$$

for $w_{m}^{l} \in \mathcal{W}_{m}^{l}$. By (6.9), there exist $C \in \mathbb{R}$ such that $\left\|W_{l}-W_{0}\right\|<C q^{\beta l}$. Furthermore, since $\alpha<\beta$, by (6.12) it follows that $\lim _{l \rightarrow \infty} q^{(\beta-\alpha) l}\left|d_{l}^{\uparrow}\right|=$ $\lim _{l \rightarrow \infty} q^{(\beta-\alpha) l}\left|d_{l}^{\downarrow}\right|=0$ and the sequences $\left\{q^{(\beta-\alpha) l}\left|d_{l}^{\uparrow}\right|\right\}$ and $\left\{q^{(\beta-\alpha) l}\left|d_{l}^{\downarrow}\right|\right\}$ are uniformly bounded. Thus,

$$
\begin{aligned}
\left\|W_{l} \widetilde{D}_{l} W_{l}-W_{0} \widetilde{D}_{l} W_{0}\right\| & =\left\|\left(W_{l}-W_{0}\right) \widetilde{D}_{l} W_{l}+W_{0} \widetilde{D}_{l}\left(W_{l}-W_{0}\right)\right\| \\
& <2\left\|W_{l}-W_{0}\right\|\left\|D_{l}\right\|<C^{\prime} q^{\alpha l},
\end{aligned}
$$

for some $C^{\prime} \in \mathbb{R}$, and $D_{1}-D \in \mathcal{l}\left(L_{q}^{\alpha}\right)$. Similarly one shows that $J_{1}-J \in \mathcal{l}\left(L_{q}^{\alpha}\right)$.

Remark 6.7. For the standard sphere, corresponding to $t=1$, the conditions (5.8) for bounded commutators of the Dirac operator with algebra elements allow more than linear dependence for the eigenvalues of $D$. In [7], the eigenvalues of $D$ were taken to be $q$-analogues of the spectrum of the classical Dirac operator of the round metric of the sphere $\mathrm{S}^{2}$; they behave like $\left|d_{l}\right| \sim q^{-l}$ for large $l$. For this family, one gets a spectral triple; this is also a particular case of our Proposition 5.1. Moreover, one has a stronger result on the real structure: it is not up to infinitesimals but the stronger relations (2.2) are satisfied.

\section{Equivariant real even spectral triple}

In this section an additional character enters the stage, the even structure. As shown in the next proposition, the existence of a grading operator determines completely the structure of the geometry $\left(\mathcal{A}\left(\mathrm{S}_{q t}^{2}\right), \mathscr{H}, D, J, \gamma\right)$.

Proposition 7.1. Let $\left(\mathcal{A}\left(\mathrm{S}_{q t}^{2}\right), \mathcal{H}, D, J, \gamma\right)$ be an equivariant real even spectral triple with real structure $J$ satisfying condition (6.1). Then the operators $\gamma, D, J$ act on the spinor bundle $W$ by

$$
\begin{aligned}
\gamma v_{m, \pm}^{l}= \pm \epsilon v_{m, \pm}^{l}, & J v_{m, \pm}^{l}=\zeta_{l}(-1)^{m+1 / 2} v_{-m, \mp}^{l}, \\
D v_{m,+}^{l}=d_{l} v_{m,-}^{l}, & D v_{m,-}^{l}=\bar{d}_{l} v_{m,+}^{l},
\end{aligned}
$$

where $d_{l} \in \mathbb{C}, \epsilon \in\{-1,1\}$, and $\zeta_{l} \in \mathbb{C}$ such that $\left|\zeta_{l}\right|=1$. 
Proof. Clearly, $\gamma \neq 1$ as otherwise the first condition in equation (2.1) is not satisfied. From $\gamma^{*}=\gamma$ and $\gamma^{2}=1$, it follows that $\gamma$ has eigenvalues \pm 1 . Since $\gamma$ commutes with all elements from the crossed product algebra $\mathcal{A}\left(\mathrm{S}_{q t}^{2}\right) \rtimes U_{q}(\mathrm{su}(2))$ and since the integrable representation of $\mathcal{A}\left(\mathrm{S}_{q t}^{2}\right) \rtimes \mathcal{U}_{q}(\mathrm{su}(2))$ on the spinor bundle $\mathcal{W}$ decomposes into two inequivalent irreducible ones on $\mathcal{M}_{-}$and $\mathcal{M}_{+}$, we conclude that $\gamma v_{m,+}^{l}=$ $\epsilon v_{m,+}^{l}$ and $\gamma v_{m,-}^{l}=-\epsilon v_{m,-}^{l}$, where $\epsilon \in\{-1,1\}$.

Recall from Lemma 6.4 that $J\left\lceil_{w_{m}^{l}}=V_{l} J_{0}\left\lceil_{w_{m}^{l}}\right.\right.$ with a unitary operator $V_{l}$ on $\mathbb{C}^{2}$. In addition, the condition $J \gamma=-\gamma J$ implies that $J$ maps $\mathcal{M}_{ \pm}$into $\mathcal{M}_{\mp}$. Thus

$$
J v_{m, \pm}^{l}=\zeta_{l, \pm} J_{0} v_{m, \mp}^{l},
$$

with complex numbers $\zeta_{l,-}$ and $\zeta_{l,+}$ such that $\left|\zeta_{l,-}\right|=\left|\zeta_{l,+}\right|=1$. From $J^{2}=-1$, we obtain $\zeta_{l,-} \bar{\zeta}_{l,+}=1$, so $\zeta_{l,-}=\zeta_{l,+}=: \zeta_{l}$.

Similarly, the condition $D \gamma=-\gamma D$ implies that $D$ maps $\mathcal{M}_{ \pm}$into $\mathcal{M}_{\mp}$. Hence, by (5.1), $D$ has the form described in (7.1).

We combine Propositions 6.5 and 7.1 to present equivariant real even spectral triples.

Proposition 7.2. Let $\gamma$ and $D$ be self-adjoint operators and $J$ be an antiunitary operator on $\mathscr{H}$ given by

$$
\gamma v_{m, \pm}^{l}= \pm v_{m, \pm}^{l}, D v_{m, \pm}^{l}=\left(c_{1} l+c_{2}\right) v_{m, \mp}^{l}, \quad J v_{m, \pm}^{l}=(-1)^{m+1 / 2} v_{-m, \mp}^{l},
$$

where $c_{1}, c_{2} \in \mathbb{R}$ with $c_{1} \neq 0$. Then $\left(\mathcal{A}\left(\mathrm{S}_{q t}^{2}\right), \mathscr{H}, D, J, \gamma\right)$ is an equivariant real even spectral triple such that the conditions (2.3) are satisfied with $d=d\left(L_{q}^{\alpha}\right)$, where $\alpha \in(0,2)$ for $t \neq 1$ and $\alpha \in(0,1)$ for $t=1$.

Proof. For $l=\frac{1}{2}, \frac{2}{3}, \ldots$, set $d_{l}^{\uparrow}=c_{1} l+c_{2}$ and $d_{l}^{\downarrow}=-\left(c_{1} l+c_{2}\right)$. Define

$$
W_{0}:=\frac{1}{\sqrt{2}}\left(\begin{array}{cc}
1 & 1 \\
-\mathrm{i} & \mathrm{i}
\end{array}\right), \quad W_{l}:=W_{0} .
$$

The restrictions of $D$ and $J$ to $W_{m}^{l}$ are then given as in (6.6) and (6.7), respectively. With the choice (7.3), the inequality (6.9) holds trivially and we can suppose that $\beta=2$ for $t \neq 1$ and $\beta=1$ for $t=1$. By Proposition $6.5,\left(\mathcal{A}\left(\mathrm{S}_{q t}^{2}\right), \mathscr{H}, D, J\right)$ yields an equivariant real spectral triple such that the conditions (2.3) are satisfied with $d=d\left(L_{q}^{\alpha}\right)$. It is obvious that $\gamma$ fulfills all the requirements of a grading operator.

Remark 7.3. Recall from Subsection 4.2 that $\mathcal{W}$ can be considered as a deformation of the classical spinor bundle. The classical spectral triple on the commutative 2sphere with its round metric is real and even, and the corresponding Dirac operator 
has eigenvalues $\pm\left(l+\frac{1}{2}\right), l=\frac{1}{2}, \frac{2}{3}, \ldots$, each with multiplicity $2 l+1$. Therefore we can regard the equivariant real even spectral triple $\left(\mathcal{A}\left(\mathrm{S}_{q t}^{2}\right), \mathcal{H}, D, J, \gamma\right)$ from Theorem 7.2 with $c_{1}=1$ and $c_{2}=\frac{1}{2}$ as an isospectral deformation of the classical spin geometry.

Remark 7.4. By perturbing both $D$ and $J$ by infinitesimals belonging to the ideal $d\left(L_{q}^{\alpha}\right)$, one produces more examples of equivariant real even spectral triples. However, those from Theorem 7.2 are distinguished by being obtained from the isospectral deformation via rescaling linearly the eigenvalues of $D$.

Finally, we prove the non-triviality of our noncommutative geometry.

Proposition 7.5. Let $F \in \mathscr{B}(\mathscr{H})$ be the sign of the Dirac operator; it is given by

$$
F v_{m, \pm}^{l}=v_{m, \mp}^{l} .
$$

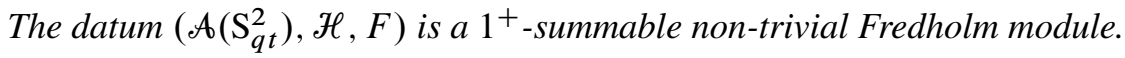

Proof. Since the chiral spin representations $\pi_{ \pm}$coincide modulo smoothing operators, the commutator $[F, x]$ is a smoothing operator for all $x \in \mathcal{A}\left(\mathrm{S}_{q t}^{2}\right)$, thus $[F, x] \in$ $\mathscr{L}^{1}(\mathscr{H})$. This shows that $\left(\mathcal{A}\left(\mathrm{S}_{q t}^{2}\right), \mathcal{H}, F\right)$ is a $1^{+}$-summable Fredholm module.

As representative of the corresponding periodic cyclic cohomology class $\mathrm{ch}^{F}$ we can take the cochain having only one component $\mathrm{ch}_{0}^{F}$ in degree 0 , given by,

$$
\operatorname{ch}_{0}^{F}(a):=\frac{1}{2} \operatorname{Tr}(\gamma F[F, a]) .
$$

The non-triviality of our Fredholm module is proved by pairing $\mathrm{ch}^{F}$ with the $K$-theory class of the projection

$$
p=\frac{1}{(2-t)\left(1+q^{2}\right)}\left(\begin{array}{cc}
1+q^{2}+x_{0}-t q^{2} & -\left(1+q^{2}\right)^{1 / 2} x_{1} \\
q\left(1+q^{2}\right)^{1 / 2} x_{-1} & 1+q^{2}-q^{2} x_{0}-t
\end{array}\right),
$$

describing line bundles over the spheres $\mathrm{S}_{q t}^{2}$ [1]. The pairing is

$$
\begin{aligned}
\left\langle\operatorname{ch}^{F},[p]\right\rangle & :=\frac{1}{2} \operatorname{Tr}_{\mathscr{H} \otimes \mathbb{C}^{2}}(\gamma F[F, p])=\frac{1-q^{2}}{2(2-t)\left(1+q^{2}\right)} \operatorname{Tr}_{\mathscr{H}}\left(\gamma F\left[F, x_{0}\right]\right) \\
& =q^{-2}\left(1-q^{2}\right)^{2} \sum_{l, m} \frac{[l-m+1][l+m]}{[2 l][2 l+2]} .
\end{aligned}
$$

For the last equality we have used the explicit formulae (4.2b) and (4.3b) for the coefficients $\alpha_{0}^{0}(l, m ; \pm)$ in the representation of $x_{0}$. The last series in (7.5) was shown in [8], Section 5.3, to be a continuous function in $q$ for $q \in[0,1)$. Since it is integer valued in the interior of this interval (being the index of a Fredholm operator), it is constant by continuity and can be computed at $q=0$. The result is $\left\langle\mathrm{ch}^{F},[p]\right\rangle=1$. 


\section{Analytic properties}

We describe now some further analytic properties of the spectral triple given in Proposition 7.2 (forgetting its real even structure) and specify, for simplicity, the constants $c_{1}=1$ and $c_{2}=1 / 2$. With this choice, $D$ is invertible. For other allowed values, the subsequent results remain valid with minor changes in the proofs and by working with $\left(1+D^{2}\right)^{-z / 2}$ instead of $|D|^{-z}$ for a not invertible $D$. We use the polar decomposition $D=F|D|$, with $F$ the sign of $D$.

Recall that the spin representation $\pi=\pi_{-} \oplus \pi_{+}$for each of the generators of the algebra $\mathcal{A}\left(\mathrm{S}_{q t}^{2}\right)$ has three terms appearing on the right-hand side of (4.1). Accordingly, we shall write $\pi\left(x_{i}\right)=x_{i}=: x_{i}^{-}+x_{i}^{0}+x_{i}^{+}, i=-1,0,1$. The operators $x_{i}^{v}$ are weighted shifts, mapping each $W_{m}^{l}$ into $W_{m+i}^{l+v}, v=-1,0,1$, which are easily seen to be bounded.

Proposition 8.1. The spectral triple $\left(\mathcal{A}\left(\mathrm{S}_{q t}^{2}\right), \mathcal{H}, D\right)$ is $2^{+}$-summable and regular.

Proof. The $2^{+}$-summability follows from the linear growth of the spectrum of $D$ (with the appropriate multiplicities). To show that the spectral triple is regular, it is enough to prove that the elements $x_{i}^{v}$ and $\left[D, x_{i}^{v}\right], v=0, \pm 1$, are in the smooth domain of the derivation $\delta(\cdot):=[|D|,(\cdot)]$. This is clear for $x_{i}^{v}$ since

$$
\left[|D|, x_{i}^{\nu}\right] v_{m, \pm}^{l}=\left(\left(l+v+\frac{1}{2}\right) \alpha_{i}^{\nu}(l, m ; \pm)-\left(l+\frac{1}{2}\right) \alpha_{i}^{\nu}(l, m ; \pm)\right) v_{m+i, \pm}^{l+v},
$$

hence $\delta\left(x_{i}^{\nu}\right)=v x_{i}^{\nu}$. As for $\left[D, x_{i}^{\nu}\right]$, we use the fact that $\delta^{k}\left(\left[D, x_{i}^{\nu}\right]\right)=\left[D, \delta^{k}\left(x_{i}^{\nu}\right)\right]=$ $v^{k}\left[D, x_{i}^{\nu}\right]$. The boundedness of the latter follows from the formulae in Proposition 4.1 giving, for instance,

$$
\left[D, x_{i}^{+}\right] v_{m, \pm}^{l}=\left(\left(l+\frac{1}{2}\right)\left(\alpha_{i}^{+}(l, m ; \pm)-\alpha_{i}^{+}(l, m ; \mp)\right)+\alpha_{i}^{+}(l, m ; \pm)\right) v_{m+i, \mp}^{l+1} .
$$

The last summand is clearly bounded while the boundedness of the other follows from the fact that $\alpha_{i}^{+}(l, m ; \pm)-\alpha_{i}^{+}(l, m ; \mp)$ is at least of order $q^{l}$ by equations (4.2) and (4.3a). Similar arguments work for $\left[D, x_{i}^{-}\right]$.

To compute the dimension spectrum, we introduce another representation of the algebra $\mathcal{A}\left(\mathrm{S}_{q t}^{2}\right)$ which is obtained from simpler operators. Let $\widehat{\mathscr{H}}$ be a Hilbert space with orthonormal basis $|l, m\rangle_{ \pm}$, where $l \in \frac{1}{2} \mathbb{Z}$ and $l+m \in \mathbb{N}_{0}$. Consider the bounded operators $\alpha, \beta \in \mathcal{B}(\hat{\mathscr{H}})$ defined by

$$
\begin{aligned}
\alpha|l, m\rangle_{ \pm} & =\sqrt{1-q^{2(l+m+1)}}\left|l+\frac{1}{2}, m+\frac{1}{2}\right\rangle_{ \pm}, \\
\beta|l, m\rangle_{ \pm} & =q^{l+m}\left|l+\frac{1}{2}, m-\frac{1}{2}\right\rangle_{ \pm} .
\end{aligned}
$$

These operators satisfy the commutation relations of $\mathcal{A}\left(\mathrm{SU}_{q}(2)\right)$, i.e.,

$$
\beta \alpha=q \alpha \beta, \quad \beta^{*} \alpha=q \alpha \beta^{*},\left[\beta, \beta^{*}\right]=0, \alpha^{*} \alpha+q^{2} \beta^{*} \beta=1, \alpha \alpha^{*}+\beta \beta^{*}=1 .
$$


The embedding of the Podleś spheres into $\mathcal{A}\left(\mathrm{SU}_{q}(2)\right)$ (see e.g. [12]) leads to a $*$-representation $\varphi: \mathcal{A}\left(\mathrm{S}_{q t}^{2}\right) \rightarrow \mathscr{B}(\hat{\mathscr{H}})$ given by

$$
\begin{aligned}
& \varphi\left(x_{1}\right)=\sqrt{1+q^{2}}\left\{\sqrt{1-t}\left(\alpha^{2}-q\left(\beta^{*}\right)^{2}\right)-t \beta^{*} \alpha\right\}, \\
& \varphi\left(x_{0}\right)=\left(1+q^{2}\right)\left\{\sqrt{1-t}\left(\alpha \beta+\beta^{*} \alpha^{*}\right)-t \beta \beta^{*}\right\}+t .
\end{aligned}
$$

Later on, we shall need the explicit expression

$$
\begin{aligned}
\left(1+q^{2}\right)^{-1} & \varphi\left(x_{0}-t\right)|l, m\rangle_{ \pm} \\
= & \sqrt{1-t} q^{l+m-1} \sqrt{1-q^{2(l+m)}}|l-1, m\rangle_{ \pm}-t q^{2(l+m)}|l, m\rangle_{ \pm} \\
& +\sqrt{1-t} q^{l+m} \sqrt{1-q^{2(l+m+1)}}|l+1, m\rangle_{ \pm} .
\end{aligned}
$$

Our Hilbert space of spinors $\mathscr{H}$ with the basis (4.4) is identified with a subspace of $\widehat{\mathscr{H}}$, that is, we consider the inclusion

$$
Q: \mathscr{H} \rightarrow \widehat{\mathscr{H}}, \quad Q v_{m, \pm}^{l}=|l, m\rangle_{ \pm}
$$

for $l \in \mathbb{N}_{0}+\frac{1}{2}$ and $|m| \leq l$. Let $P: \hat{\mathscr{H}} \rightarrow \mathscr{H}$ be the adjoint map of $Q$, i.e.,

$$
P|l, m\rangle_{ \pm}=v_{m, \pm}^{l} \text { for } l \in \mathbb{N}_{0}+\frac{1}{2} \text { and }|m| \leq l, \quad P|l, m\rangle_{ \pm}=0 \text { otherwise. }
$$

Our Dirac operator $D$ on $\mathscr{H}$ is the "restriction" of the self-adjoint operator $D^{\prime}$ on $\widehat{\mathscr{H}}$ determined by

$$
D^{\prime}|l, m\rangle_{ \pm}=\left(l+\frac{1}{2}\right)|l, m\rangle_{\mp},
$$

in the sense that $D P=P D^{\prime}, Q D=D^{\prime} Q$. The same holds for $\left|D^{\prime}\right|$ and for its sign $F^{\prime}:=D^{\prime}\left|D^{\prime}\right|^{-1}$. The subspace $Q \mathscr{H}$ is not invariant for the representation $\varphi$. However, we can sandwich $\varphi$ between $Q$ and $P$ thus obtaining a $*$-linear map

$$
\tilde{\varphi}: \mathcal{A}\left(\mathrm{S}_{q t}^{2}\right) \rightarrow \mathcal{B}(\mathcal{H}), \quad \tilde{\varphi}(a)=P \varphi(a) Q,
$$

that has the following approximation property.

Lemma 8.2. The operator $a-\tilde{\varphi}(a)$ is a smoothing operator on $\mathcal{H}$ for all $a \in \mathcal{A}\left(\mathrm{S}_{q t}^{2}\right)$.

Proof. Observe that if $T: \hat{\mathscr{H}} \rightarrow \mathscr{H}$ is a matrix of rapid decay (in our fixed bases), then so are $a T \varphi(b)$, where $a, b \in \mathcal{A}\left(\mathrm{S}_{q t}^{2}\right)$, and $T Q: \mathcal{H} \mapsto \mathcal{H}$. Using arguments similar to the ones in the proof of Lemma 4.4, for the generators $x_{i}, i=-1,0,1$, one verifies by direct computations that $x_{i} P-P \varphi\left(x_{i}\right)$ yields a rapid decay matrix. Then the lemma follows from the identity

$$
a b P-P \varphi(a b)=a(b P-P \varphi(b))+(a P-P \varphi(a)) \varphi(b)
$$

by applying $P Q=\mathrm{id}_{\mathscr{H}}$ and by the above observations. 
The next proposition is the main result of this section.

Proposition 8.3. The dimension spectrum is $\Sigma=\{1,2\}$.

Proof. Let $\Psi^{0}$ be the algebra generated by $\mathcal{A}\left(\mathrm{S}_{q t}^{2}\right)$, by $[D, a]$ for all $a \in \mathcal{A}\left(\mathrm{S}_{q t}^{2}\right)$ and by iterated applications of the derivation $\delta$ (cf. Section 2). Let $\mathcal{C}$ be the $*$-algebra (of bounded operators on $\widehat{\mathscr{H}}$ ) generated by $\alpha, \beta, \alpha^{*}, \beta^{*}$ and $F$. By Lemma 8.2, $\mathcal{A}\left(\mathrm{S}_{q t}^{2}\right) \subset P \mathscr{C} Q+\mathrm{OP}^{-\infty}$. Note that

$$
\begin{array}{lll}
{\left[F^{\prime}, \alpha\right]=0,} & {\left[D^{\prime}, \alpha\right]=\frac{1}{2} \alpha F^{\prime},} & {\left[\left|D^{\prime}\right|, \alpha\right]=\frac{1}{2} \alpha,} \\
{\left[F^{\prime}, \beta\right]=0,} & {\left[D^{\prime}, \beta\right]=\frac{1}{2} \beta F^{\prime},} & {\left[\left|D^{\prime}\right|, \alpha\right]=\frac{1}{2} \beta .}
\end{array}
$$

Thus $P \subset Q$ is invariant under application of $\delta$ and $[D,(\cdot)]$ and it follows that $\Psi^{0} \subset P \mathscr{C} Q+\mathrm{OP}^{-\infty}$.

We shall compute the singularities of zeta functions associated to the monomials $S:=P \alpha^{n} \beta^{j}\left(\beta^{*}\right)^{k} Q F$ and $T:=P \alpha^{n} \beta^{j}\left(\beta^{*}\right)^{k} Q$, where $n \in \mathbb{Z}$ and $j, k \in \mathbb{N}_{0}$ and we employ the notation $\alpha^{n}:=\left(\alpha^{*}\right)^{|n|}$ for $n<0$. From the commutation relations of $\alpha$ and $\beta$, it is clear that these monomials span $P \mathcal{C} Q$.

Firstly, note that the $\zeta$ function associated with a bounded off-diagonal operator is identically zero in the half-plane $\operatorname{Re} z>2$ and so is its holomorphic extension to the entire complex plane. This is the case for the monomials $S$ due to the presence of $F$. The other monomials $T$ shift the index $l$ by $(n+j-k) / 2$ and the index $m$ by $(n-j+k) / 2$ and therefore are also off-diagonal operators unless these shifts are zero, which happens when $n=0$ and $j=k$. Hence only monomials $T=$ $P \beta^{k}\left(\beta^{*}\right)^{k} Q=P\left(\beta \beta^{*}\right)^{k} Q$ contribute to the dimension spectrum.

For $k=0, T=\mathrm{id}$, and the corresponding zeta function is

$$
\zeta_{\text {id }}(z)=\sum_{l+\frac{1}{2} \in \mathbb{N}} \sum_{l+m=0}^{2 l} \sum_{r= \pm}\left(l+\frac{1}{2}\right)^{-z}=4 \zeta(z-1),
$$

where $\zeta(z)$ is the Riemann zeta function, meromorphic in $\mathbb{C}$ with a simple pole at 1 and with residue 1 . Since id $\in \Psi^{0}$, this shows that $2 \in \Sigma$.

When $k>0, T=P\left(\beta \beta^{*}\right)^{k} Q$. So $T v_{m, \pm}^{l}=q^{2 k(l+m)} v_{m, \pm}^{l}$ and the associated zeta function is

$$
\begin{aligned}
\zeta_{T}(z) & =\sum_{l+\frac{1}{2} \in \mathbb{N}} 2\left(l+\frac{1}{2}\right)^{-z} \sum_{l+m=0}^{2 l}\left(q^{2 k}\right)^{l+m}=\sum_{l+\frac{1}{2} \in \mathbb{N}} 2\left(l+\frac{1}{2}\right)^{-z} \frac{1-q^{2 k(2 l+1)}}{1-q^{2 k}} \\
& =\frac{2}{1-q^{2 k}} \zeta(z)+\text { holomorphic function. }
\end{aligned}
$$

Therefore $\Sigma$ may contain, besides 2 , at most the additional point 1 . We still have to check that $1 \in \Sigma$ since the algebra $P \mathcal{C} Q$ is strictly larger than $\Psi^{0}$. For this, we take 
$a \in \mathcal{A}\left(\mathrm{S}_{q t}^{2}\right)$, where $\left(1+q^{2}\right)^{2} a=\left(x_{0}-t\right)^{2}=\tilde{\varphi}\left(\left(x_{0}-t\right)^{2}\right)+$ smoothing terms. Then, using (8.1), we get (modulo holomorphic functions)

$$
\begin{aligned}
\zeta_{a}(z) & \sim 2 \sum_{l+\frac{1}{2} \in \mathbb{N}}\left(l+\frac{1}{2}\right)^{-z} \sum_{k=0}^{2 l}\left\{t^{2} q^{4 k}+(1-t) b\left\{\left(1+q^{-2}\right) q^{2 k}-\left(q^{2}+q^{-2}\right) q^{4 k} b\right\}\right\} \\
& \sim 2 \frac{1+(1-t)^{2}}{1-q^{4}} \zeta(z),
\end{aligned}
$$

and $\operatorname{Res}_{z=1} \zeta_{a}(z)=2 \frac{1+(1-t)^{2}}{1-q^{4}} \neq 0$ for all $t \in[0,1]$. This shows that $\Sigma=\{1,2\}$.

Let $\mathcal{A}\left(\mathrm{S}^{1}\right)$ denote the polynomial $*$-algebra in one variable $\lambda$, with $\lambda \bar{\lambda}=1$. For $t \neq 1$, we have $*$-algebra morphisms $\sigma_{t}: \mathcal{A}\left(\mathrm{S}_{q t}^{2}\right) \rightarrow \mathcal{A}\left(\mathrm{S}^{1}\right)$ given by the "classical points' (3.3),

$$
\sigma_{t}\left(x_{0}\right)=t, \quad \sigma_{t}\left(x_{1}\right)=\sqrt{\left(1+q^{2}\right)(1-t)} \lambda .
$$

For $t=1$, let $\sigma_{1}: \mathcal{A}\left(\mathrm{S}_{q 1}^{2}\right) \rightarrow \mathbb{C}$ be the $*$-algebra morphism given by the "classical point' (3.2),

$$
\sigma_{1}\left(x_{0}\right)=1, \quad \sigma_{1}\left(x_{1}\right)=0 .
$$

Proposition 8.4. The top residue of the zeta-type function $\zeta_{a}(z):=\operatorname{Tr}_{\mathscr{H}}\left(a|D|^{-z}\right)$, with $a \in \mathcal{A}\left(\mathrm{S}_{q t}^{2}\right)$, is given by

$$
\operatorname{Res}_{z=2} \zeta_{a}(z)=-\frac{2 \mathrm{i}}{\pi} \int_{\mathrm{S}^{1}} \sigma_{t}(a) \frac{\mathrm{d} \lambda}{\lambda}, \quad 0 \leq t \leq 1 .
$$

For $t=1, \sigma_{1}(a) \in \mathbb{C}$ and equation (8.3) simplifies to $4 \sigma_{1}(a)$.

Proof. It is sufficient to prove (8.3) for the basis elements $\left(x_{0}-t\right)^{j} x_{1}^{k}, j \in \mathbb{N}_{0}$ and $k \in \mathbb{Z}$, and then extend it to $\mathcal{A}\left(\mathrm{S}_{q t}^{2}\right)$ by linearity. We use again the notation $x_{1}^{k}:=\left(x_{1}^{*}\right)^{|k|}$ if $k<0$.

Since $\sigma_{t}\left(\left(x_{0}-t\right)^{j} x_{1}^{k}\right) \propto \delta_{j 0}(1-t)^{k / 2} \lambda^{k}$, the right-hand side of equation (8.3) is zero unless $j=k=0$. We next show that the left hand side of (8.3) also vanishes unless $j=k=0$. When $j=k=0$, the relation $\zeta_{\text {id }}(z)=4 \zeta(z-1)$ fixes the normalization constant.

Now $\left(x_{0}-t\right)^{j} x_{1}^{k}$ is off-diagonal if $k \neq 0$ since it shifts the index $m$ by $k$. It remains to prove that $\zeta_{\left(x_{0}-t\right)^{j}}(z)=\zeta_{\widetilde{\varphi}\left(\left(x_{0}-t\right)^{j}\right)}(z)+$ holomorphic function has no singularity in $z=2$. For $j \neq 0, \widetilde{\varphi}\left(\left(x_{0}-t\right)^{j}\right)$ satisfies the inequality

$$
\left|\left(v_{m, \pm}^{l}, \tilde{\varphi}\left(\left(x_{0}-t\right)^{j}\right) v_{m, \pm}^{l}\right)\right| \leq c_{j} q^{l+m},
$$

for some positive constants $c_{j}$ 's. From this inequality, we deduce that $\zeta_{\widetilde{\varphi}\left(\left(x_{0}-t\right)^{j}\right)}(z)$ is a convergent series for all $z$ with $\operatorname{Re} z>1$. In particular, it is finite for $z=2$. 
A natural application of the analysis in this section concerns the construction of a local representative of the cocycle $\mathrm{ch}^{F}$, which appeared in the proof of Proposition 7.5. For our case, Theorem II.3 of Connes-Moscovici [4] states that $\mathrm{ch}^{F}$ is cohomologous to the periodic cyclic cocycle with two components $\left(\phi_{0}, \phi_{2}\right)$, given by

$$
\begin{gathered}
\phi_{0}\left(a_{0}\right)=\operatorname{Res}_{z=0} z^{-1} \operatorname{Tr}\left(\gamma a_{0}|D|^{-2 z}\right), \\
\phi_{2}\left(a_{0}, a_{1}, a_{2}\right)=\operatorname{Res}_{z=0} \operatorname{Tr}\left(\gamma a_{0}\left[D, a_{1}\right]\left[D, a_{2}\right]|D|^{-2(z+1)}\right) .
\end{gathered}
$$

Smoothing operators do not contribute to $\phi_{2}$. Since $[D, a]=\delta \widetilde{\varphi}(a) F+\mathrm{OP}^{-\infty}$, with the map $\widetilde{\varphi}$ given in (8.2), we can rewrite $\phi_{2}$ as,

$$
\phi_{2}\left(a_{0}, a_{1}, a_{2}\right)=\operatorname{Res}_{z=0} \operatorname{Tr}\left(\gamma \tilde{\varphi}\left(a_{0}\right) \delta \tilde{\varphi}\left(a_{1}\right) \delta \tilde{\varphi}\left(a_{2}\right)|D|^{-2(z+1)}\right) .
$$

Now $\gamma$ is traceless and the remaining operators are diagonal in the spin index " \pm "; thus $\phi_{2}$ is identically zero. Moreover, $\operatorname{Tr}\left(\gamma a_{0}|D|^{-2 z}\right)$ is holomorphic for $\operatorname{Re} z>1$ where it coincides with the function $\psi(z):=\frac{1}{2} \operatorname{Tr}\left(\gamma F\left[F, a_{0}\right]|D|^{-2 z}\right)$, which is holomorphic on all $\mathbb{C}$, being $\left[F, a_{0}\right] \in \mathrm{OP}^{-\infty}$. Hence,

$$
\phi_{0}\left(a_{0}\right)=\operatorname{Res}_{z=0} z^{-1} \psi(z)=\psi(0)=\operatorname{ch}_{0}^{F}\left(a_{0}\right),
$$

with $\mathrm{ch}_{0}^{F}$ given by equation (7.4).

This shows that, due to the low summability of the Fredholm module, only the lowest (non-local) component of the periodic cyclic cochain of Theorem II.3 of [4] is different from zero, and equals $\mathrm{ch}_{0}^{F}$. This fact was already shown in [8] for the equatorial Podleś sphere.

\section{Final remarks}

A crucial ingredient of our analysis was the approximation of $\pi\left(x_{i}\right)=\left(\pi_{-\frac{1}{2}} \oplus \pi_{\frac{1}{2}}\right)\left(x_{i}\right)$ by the operators $z_{i}$ defined in (4.5). The proof of Lemma 4.4 shows that a similar approximation holds true for the representation $\pi_{-N} \oplus \pi_{N}$ on $\mathcal{M}_{-N} \oplus \mathcal{M}_{N}$, for any $N \in \frac{1}{2} \mathbb{N}$. A careful inspection of the subsequent proofs shows that all results are still valid and yield corresponding spectral triples with real structure $J$ satisfying $J^{2}=(-1)^{N}$, and the pairing of the associated Fredholm module with the same projection as in Corollary 7.5 giving the value $2 N$. In the classical case $q=1$, these generalized examples correspond to the quasi-spectral triples studied in [15].

Acknowledgements. It is a pleasure to thank J. Varilly for helpful comments. F. D'A., L.D. and G. L. thank ESI in Vienna for hospitality. L. D. was partially supported by the European Union Host Fellowship for the Transfer of Knowledge Program 'Noncommutative Geometry and Quantum Groups' at Warsaw University. E. W. was supported by the DFG-grant Wa 1698/2-1. 


\section{References}

[1] T. Brzeziński and S. Majid, Quantum geometry of algebra factorisations and coalgebra bundles. Comm. Math. Phys. 213 (2000), 491-521. Zbl 0979.58002 MR 1785427

[2] A. Connes, Noncommutative geometry. Academic Press, San Diego, CA, 1994. Zbl 0818.46076 MR 1303779

[3] A. Connes, Gravity coupled with matter and the foundation of non-commutative geometry. Comm. Math. Phys. 182 (1996), 155-176. Zbl 0881.58009 MR 1441908

[4] A. Connes and H. Moscovici, The local index formula in noncommutative geometry. Geom. Funct. Anal. 5 (1995), 174-243. Zbl 0960.46048 MR 1334867

[5] L. Dạbrowski, G. Landi, M. Paschke, and A. Sitarz, The spectral geometry of the equatorial Podleś sphere. C. R. Acad. Sci. Paris Sér. I Math. 340 (2005), 819-822. Zbl 1072.58002 MR 2139894

[6] L. Dạbrowski, G. Landi, A. Sitarz, W. van Suijlekom, and J. C. Várilly, The Dirac operator on $S U_{q}$ (2). Comm. Math. Phys. 259 (2005), 729-759. Zbl 1090.58504 MR 2174423

[7] L. Dạbrowski and A. Sitarz, Dirac operator on the standard Podleś quantum sphere. In Noncommutative geometry and quantum groups (Warsaw, 2001), Banach Center Publ. 61, Polish Acad. Sci., Warsaw 2003, 49-58. Zbl 1061.58004 MR 2024421

[8] F. D'Andrea and L. Da̧browski, Local index formula on the equatorial Podleś sphere. Lett. Math. Phys. 75 (2006), 235-254. Zbl 05068077 MR 2211030

[9] P. M. Hajac and S. Majid, Projective module description of the $q$-monopole. Comm. Math. Phys. 206 (1999), 247-264. Zbl 0936.46052 MR 1722149

[10] A. U. Klimyk and K. Schmüdgen, Quantum groups and their representations. Texts Monogr. Phys., Springer-Verlag, Berlin 1997. Zbl 0891.17010 MR 1492989

[11] E. F. Müller and H.-J. Schneider, Quantum homogeneous spaces with faithfully flat module structures. Israel J. Math. 111 (1999), 157-190. Zbl 1001.17015 MR 1710737

[12] P. Podleś, Quantum spheres. Lett. Math. Phys. 14 (1987), 193-202. Zbl 0634.46054 MR 919322

[13] K. Schmüdgen and E. Wagner, Representations of cross product algebras of Podleś quantum spheres. Preprint, 2003; arXiv:math.QA/0305309

[14] A. Sitarz, Equivariant spectral triples. In Noncommutative geometry and quantum groups (Warsaw, 2001), Banach Center Publ. 61, Polish Acad. Sci., Warsaw 2003, 231-263. Zbl 1061.58007 MR 2024433

[15] A. Sitarz, Quasi-Dirac operators on the sphere. Preprint, 2006; arXiv:math-ph/0602030

Received June 17, 2006

Ludwik Dąbrowski, Francesco D’Andrea, Scuola Internazionale Superiore di Studi Avanzati, Via Beirut 2-4, 34014 Trieste, Italy

E-mail: dabrow@sissa.it, dandrea@sissa.it

Giovanni Landi, Elmar Wagner, Dipartimento di Matematica e Informatica, Università di Trieste, Via Valerio 12/1, 34127 Trieste, Italy

E-mail: landi@univ.trieste.it, wagner@dmi.units.it 